\title{
Aperiodic sampled-data MPC strategy for LPV systems ${ }^{\star}$
}

\author{
Alessandra H. K. Palmeira ${ }^{\mathrm{a}}$, João M. Gomes da Silva Jr ${ }^{\mathrm{a}, *}$, Jeferson V. Flores ${ }^{\mathrm{a}}$, Alexandre Seuret ${ }^{\mathrm{b}}$ \\ ${ }^{a}$ UFRGS, School of Engineering, Porto Alegre, Brazil \\ ${ }^{b} L A A S-C N R S$, Université de Toulouse, CNRS, UPS, Toulouse, France
}

\begin{abstract}
This paper addresses the design of a sampled-data model predictive control (MPC) strategy for linear parameter-varying (LPV) systems. A continuous-time prediction model, which takes into account that the samples are not necessarily periodic and that plant parameters continuously vary with time, is considered. Moreover, it is explicitly assumed that the value of the parameters used to compute the optimal control sequence is measured only at the sampling instants. The MPC approach proposed by Kothare et al. [1], where the basic idea consists in solving an infinite horizon guaranteed cost control problem at each sampling time using linear matrix inequalities (LMI) based formulations, is adopted. In this context, conditions for computing a sampled-data stabilizing LPV control law that provides a guaranteed cost for a quadratic performance criterion under input saturation are derived. These conditions are obtained from a parameter-dependent looped-functional and a parameter-dependent generalized sector condition. A strategy that consists in solving convex optimization problems in a receding horizon policy is therefore proposed. It is shown that the proposed strategy guarantees the feasibility of the optimization problem at each step and leads to the asymptotic stability of the origin. The conservatism reduction provided by the proposed results, with respect to similar ones in the literature, is illustrated through numerical examples.
\end{abstract}

Keywords: linear parameter-varying systems; sampled-data control; model predictive control; guaranteed cost control; constrained control; input saturation.

\section{Introduction}

Model predictive control (MPC) has received a lot of attention from the control community over the last years, both in the contexts of theoretical developments and practical applications [1-3]. The MPC strategy consists basically in solving an on-line optimization problem over a finite/infinite prediction horizon at each sampling instant and in applying the first element of the optimal control sequence until the next sampling occurs. The prediction of the system variables is

${ }^{\star}$ J.V. Flores and J.M. Gomes da Silva Jr. are also supported by the Conselho Nacional de Desenvolvimento Científico e Tecnológico - Brazil (CNPq) - Grants 422992/2016-0, 306223/2018-0 and 307449/2019-0. This study was financed in part by the Coordenação de Aperfeiçoamento de Pessoal de Nível Superior - Brazil (CAPES) Finance Code 001 (PROEX and STICAmSud programs).

${ }^{*}$ Corresponding author.

Email addresses: kimura.palmeira@ufrgs.br (Alessandra H. K. Palmeira), jmgomes@ufrgs.br (João M. Gomes da Silva Jr), jeferson.flores@ufrgs.br (Jeferson V. Flores), aseuret@laas.fr (Alexandre Seuret) 
made considering a model of the controlled process. The optimization problem is then repeated in a receding horizon policy whenever a new measurement (of the states and/or outputs) is available. This kind of control strategy has been shown to efficiently deal with performance issues and with state and control constraints (see for instance [2-5] for general overviews on MPC).

Linear parameter-varying (LPV) systems have been successfully used to model systems with parametric varying uncertainties. Moreover, some nonlinear systems can also be cast in the socalled quasi-LPV form by an appropriate conversion of the nonlinearities in the product of states and varying parameters [6]. Several practical applications of the LPV modeling can be found, for instance, in [7]. Hence, LPV models can be used in MPC strategies to take into account parameter uncertainties and nonlinear effects. A relevant approach in this context is the one originally proposed by Kothare et al. [1]. It basically consists in solving an infinite horizon guaranteed cost control problem, based on a linear matrix inequalities (LMI) framework, and then repeat the procedure at each sampling instant using the measurement of the current state. It is shown that this procedure guarantees feasibility, respect to control constraints and asymptotic stability, provided that the optimization problem is feasible at the initial instant, i.e. if there exists a robust stabilizing state feedback control law which ensures that the initial state is included in the basin of attraction of the closed-loop system origin under constrained control. This approach has been further applied and developed in [8-11].

It should be pointed out that all the references in the previous paragraph consider discretetime LPV models. A first issue in this case regards the determination of an LPV discrete-time model from an actual continuous-time system. This is not an easy task since exact discretization techniques (as the one for standard linear systems) cannot be applied [12]. The second issue lies in the implicit assumption that the plant parameters are supposed to be constant between two successive sampling instants. Note that this assumption is in general not realistic, since the plant is a continuous-time system and the time-varying parameters evolve continuously between two successive sampling instants. In addition, motivated by the development of networked and embedded control systems, a special attention has been paid to the case of aperiodic sampling [13], i.e. when the time between two sampling instants is not necessarily constant. To cope with these issues, it is more appropriate to address closed-loop stability and performance considering a sampled-data framework, where continuous-time evolution of the plant under a control signal updated only at the sampling instants is explicitly taken into account $[13,14]$. In this context, the problem of MPC for LPV systems considering a sampled-data framework is addressed in [15, 16]. In these references, the MPC strategy of Kothare et al. [1] is employed and a time-delay approach (similar to those proposed in [17-19]) is used to cope with the sampled-data problem.

Motivated by the aforementioned issues, we focus here on the problem of sampled-data MPC for LPV systems. It is explicitly assumed that the parameters and the plant states evolve in continuoustime, while their measurements to update the control signal are taken only at the sampling instants. We adopt the MPC strategy proposed by Kothare et al. [1], where an infinite guaranteed cost control problem is solved at each sampling time, using an LMI-based formulation. With this aim, we first derive LMI conditions for computing a sampled-data stabilizing LPV control law aiming at providing a guaranteed cost for an infinite horizon quadratic criterion under input saturation. Thus, a strategy that consists in solving convex optimization problems in a receding horizon policy is proposed. It is shown that this strategy leads to the feasibility of the optimization problem at each step and guarantees the asymptotic stability of the origin.

The main contributions of this paper and the differences with respect to previous literature are summarized below: 
- In most works following Kothare's approach, a formulation in the discrete-time framework (e.g. $[8-11,20]$ ) is considered. In this case, it is implicitly assumed that the parameters are constant between two samples and that the sampling is periodic. Our approach considers directly continuous-time LPV models, which allows to cope with the aperiodic sampling case and the fact that plant parameters can vary between two successive sampling instants.

- Based on the information about the bounds on the parameters variation rate, differently from [15], we propose a model that explicitly captures the mismatch between the continuous-time plant parameter and its last sampled value used for control purposes. Moreover, to derive the stabilization conditions, we consider a looped-functional. In this context, the approach originally proposed in $[21,22]$ is extended to consider parameter-dependent looped-functionals as done in [23]. In particular, differently from classical Lyapunov-Krasovskii functionals (considered for instance in [15-18]), looped-functionals are not required to be positive definite. These ingredients allow the reduction of conservatism (in terms of the guaranteed cost, maximal allowable sampling interval and stability region), when compared to the approaches considered in $[15,16]$.

- The problem of sampled-data guaranteed cost control of LPV systems under input saturation (control constraints) is formalized and conditions to solve it in a regional stability context are stated. Provided that the initial state belongs to an admissible set, described as the level set of a parameter-dependent quadratic function, it is shown that the trajectories of the sampled-data system under saturating control inputs converge asymptotically to the origin, while a bound on the quadratic performance criterion is ensured. In particular, to derive these results, a parameter-dependent version of the generalized sector condition proposed in [24] is applied. Differently from most works following the Kothare's approach, which consider saturation avoidance conditions, our results allow to deal with the effective saturation of the control signal and thus to take into account the possible nonlinear behavior of the closed-loop system.

- The MPC algorithm is proposed based on the conditions derived to solve the sampled-data guaranteed cost control problem under input saturation. Provided that the initial state belongs to an admissible set, which can also be seen as an estimate of the region of attraction of the origin, we formally show that the proposed algorithm guarantees the feasibility of the optimization problem at each sampling instant and the asymptotic stability of the origin.

This paper is organized as follows. The problem set up is presented in Section 2. Conditions to solve a sampled-data guaranteed cost problem through a saturating state feedback control law are derived in Section 3. Based on these conditions and an associated LMI-based optimization problem, in Section 4 the proposed MPC strategy is formulated and results regarding feasibility and the guarantee of asymptotic stability are provided. Numerical examples are presented in Section 5. Some concluding remarks and future directions of research are pointed out in Section 6 .

Notations. Throughout the article, sets $\mathbb{N}, \mathbb{R}^{+}, \mathbb{R}^{n}, \mathbb{R}^{n \times n}$ and $\mathbb{S}^{n}$ denote respectively the set of nonnegative integers, nonnegative scalars, $n$-dimensional vectors, $n \times n$ matrices and symmetric matrices of $\mathbb{R}^{n \times n}$. For a given positive scalar $T$, define $\mathscr{C}_{[0, T]}^{n}$ as the set of continuous functions from an interval $[0, T]$ to $\mathbb{R}^{n}$. The union set of continuous functions with support in a certain range is defined as $\mathbb{K}_{\left[\mathscr{T}_{1}, \mathscr{T}_{2}\right]}^{n}=\cup_{T \in\left[\mathscr{T}_{1}, \mathscr{T}_{2}\right]}\left\{\mathscr{C}_{[0, T]}^{n}\right\} .\|\cdot\|$ stands for the Euclidean norm of a vector. For $P \in \mathbb{S}^{n}$, $P>0$ means that $P$ is positive definite. He $\{A\}$ refers to $A+A^{\prime}$, symbols $I$ and 0 represent the identity 
and the zero matrices of appropriate dimension. $\operatorname{Co}\{\cdot\}$ stands for a convex hull. For a polytope $\mathscr{B}, \operatorname{Ver}(\mathscr{B})$ denotes the set of its vertices. $x\left(t_{k+s} \mid t_{k}\right)$ means the predicted value of $x\left(t_{k+s}\right)$ based on information available at at time $t_{k}$, for $s=0,1, \ldots, \infty$. $\otimes$ denotes Kronecker product and $\Lambda(v)$ is a shortcut for $v \otimes I$. $A_{(i)}$ and $x_{(i)}$ represent the $i$-th line of the matrix $A$ and $i$-th element of the vector $x$, respectively. For a square matrix $A, \lambda_{\max }(A)$ denotes its largest eigenvalue. $\mathscr{E}(P, c)$ denotes the ellipsoidal set $\mathscr{E}(P, c)=\left\{x \in \mathbb{R}^{n} ; x^{\prime} P x \leq c\right\}$, with $P=P^{\prime}>0, c>0$, and $\partial \mathscr{E}(P, c)$ denotes its boundary. Define $\|x(\theta)\|_{Z}^{2}=x^{\prime}(\theta) Z x(\theta)$, with $Z=Z^{\prime} \geq 0$.

\section{Problem Formulation}

\subsection{Description of the LPV System}

Consider a continuous-time LPV system with saturating sampled-data control inputs given by

$$
\begin{aligned}
& \dot{x}(t)=A(\sigma(t)) x(t)+\operatorname{Bsat}(u(t)), \\
& u(t)=u\left(t_{k}\right), \quad \forall t \in\left[t_{k}, t_{k+1}\right),
\end{aligned}
$$

where $x \in \mathbb{R}^{n}, u \in \mathbb{R}^{m}$ represent the state and the input vectors, respectively. $\sigma(t) \in \mathbb{R}^{N}$ is the vector of time-varying parameters with $A: \mathbb{R}^{N} \rightarrow \mathbb{R}^{n \times n}$ and $B \in \mathbb{R}^{n \times m}$. The sequence of sampling time instants $\left\{t_{k}\right\}_{k \in \mathbb{N}}$, with $t_{k} \in \mathbb{R}^{+}$for $k \in \mathbb{N}$, is such that $\bigcup_{k \in \mathbb{N}}\left[t_{k}, t_{k+1}\right)=[0,+\infty)$. The difference between two successive sampling instants is denoted by $T_{k}=t_{k+1}-t_{k}$ and it is assumed that there exist $\mathscr{T}_{1}$ and $\mathscr{T}_{2}$ in $\mathbb{R}^{+}$such that $0<\mathscr{T}_{1} \leq T_{k} \leq \mathscr{T}_{2}$. The particular case $T_{k}=\mathscr{T}_{1}=\mathscr{T}_{2}$, for all $k \in \mathbb{N}$, corresponds to a periodic sampling strategy. During any sampling interval $\left[t_{k}, t_{k+1}\right), u(t)$ is kept constant by means of a zero-order hold $(\mathrm{ZOH})$.

The saturation function is component-wise defined as follows:

$$
\operatorname{sat}_{(i)}(u)=\operatorname{sign}\left(u_{(i)}\right) \min \left\{|u|_{(i)}, \bar{u}_{(i)}\right\},
$$

for $i=1, \ldots, m$, where $\pm \bar{u}_{(i)}$ are the symmetric bounds on the $i$ th input signal.

We assume that each component of $\sigma(t)$, i.e. each time-varying parameter, is continuously differentiable and bounded both in amplitude and rate as follows:

$$
\begin{aligned}
& \underline{\sigma}_{(j)} \leq \sigma_{(j)}(t) \leq \bar{\sigma}_{(j)}, \quad j=1, \ldots, N . \\
& \underline{\xi}_{(j)} \leq \dot{\sigma}_{(j)}(t) \leq \bar{\xi}_{(j)}, \quad j=1,
\end{aligned}
$$

In other words, $\sigma(t)$ and $\dot{\sigma}(t)$ belong to the following convex polytopes with $2^{N}$ vertices in $\mathbb{R}^{N}$ :

$$
\begin{aligned}
\sigma(t) \in \mathscr{B}_{\sigma} & =\operatorname{Co}\left\{v_{1}, v_{2}, \ldots, v_{2^{N}}\right\} \\
\dot{\sigma}(t) \in \mathscr{B}_{\dot{\sigma}} & =\operatorname{Co}\left\{\eta_{1}, \eta_{2}, \ldots, \eta_{2^{N}}\right\}
\end{aligned}
$$

where $v_{j}$ and $\eta_{j} \in \mathbb{R}^{N}, j=1, \ldots, 2^{N}$, denote the vertices of $\mathscr{B}_{\sigma}$ and $\mathscr{B}_{\dot{\sigma}}$, respectively.

Matrix $A(\sigma(t))$ is assumed to be affine with respect to $\sigma(t)$ and can be generically represented as follows:

$$
A(\sigma(t))=A_{0}+\sum_{j=1}^{N} \sigma_{(j)}(t) A_{j}=\left(A_{0}+\mathbf{A}[\sigma(t) \otimes I]\right)=A_{0}+\mathbf{A} \Lambda(\sigma(t))
$$

with $A_{j} \in \mathbb{R}^{n \times n}$ being constant matrices for $j=0, \ldots, N, \mathbf{A} \triangleq\left[A_{1} \ldots A_{N}\right]$, and $\Lambda(v)$ being a shortcut for $v \otimes I$. 


\subsection{Guaranteed Cost Sampled-data LPV Control Problem}

As a control objective, consider the minimization of an upper bound $\gamma_{k}$ to the following quadratic cost function

$$
J\left(t_{k}, \infty\right)=\int_{t_{k}}^{\infty}\left(\|x(\theta)\|_{Z}^{2}+\|\operatorname{sat}(u(\theta))\|_{H}^{2}\right) d \theta=\int_{t_{k}}^{\infty}\left(x^{\prime}(\theta) Z x(\theta)+\operatorname{sat}(u(\theta))^{\prime} H \operatorname{sat}(u(\theta))\right) d \theta,
$$

where $Z \geq 0$ and $H>0$ are symmetric weighting matrices with appropriate dimensions. We consider that $Z=\bar{Z}^{\prime} \bar{Z}$, with $\bar{Z} \in \mathbb{R}^{q \times n}$ and $q \leq n$.

Assuming that the parameters can be measured, we consider a sampled-data LPV state feedback control law, i.e. the control signal is assumed to be computed from the values of $\sigma(t)$ and $x(t)$ obtained at the sampling instants $t=t_{k}$. In particular, we consider an LPV state feedback control law given as follows:

$$
u(t)=K\left(\sigma\left(t_{k}\right)\right) x\left(t_{k}\right), \forall t \in\left[t_{k}, t_{k+1}\right), \forall k \in \mathbb{N},
$$

where $K\left(\sigma\left(t_{k}\right)\right) \in \mathbb{R}^{m \times n}$ is an LPV gain matrix that depends affinely on the parameters measured at sampling instant $t=t_{k}$, i.e.:

$$
K\left(\sigma\left(t_{k}\right)\right)=K_{0}+\sum_{j=1}^{N} \sigma_{(j)}\left(t_{k}\right) K_{j}=K_{0}+\mathbf{K} \Lambda\left(\sigma\left(t_{k}\right)\right)
$$

with $K_{j} \in \mathbb{R}^{m \times n}$ being constant matrices for $j=0, \ldots, N$ and $\mathbf{K}=\left[\begin{array}{lll}K_{1} & \ldots K_{N}\end{array}\right]$.

Note that $u(t)$ is supposed to be kept constant over the interval $\left[t_{k}, t_{k+1}\right)$ with the value computed from $\sigma$ and $x$ measured at the instant $t=t_{k}$. At each sampling time, the gain matrix $K\left(\sigma\left(t_{k}\right)\right)$ is therefore updated based on the new measurement of the time-varying parameters. It is worth noticing that the plant and parameters evolve in continuous-time, while the controller signal (5) is computed and updated only at the sampling instants $t=t_{k}$. To take this fact into account, the scheduling function $\sigma(t)$ can be decomposed as follows [25]:

$$
\sigma(t)=\sigma\left(t_{k}\right)+\left(\sigma(t)-\sigma\left(t_{k}\right)\right)=\sigma\left(t_{k}\right)+\delta_{k}(t), \forall t \in\left[t_{k}, t_{k+1}\right),
$$

where $\delta_{k}(t)$ denotes the possible variation of $\sigma(t)$ in the intersampling interval. Assuming that $\bar{\xi}_{(j)} \geq 0, \underline{\xi}_{(j)} \leq 0, \dot{\delta}_{k}(t)=\dot{\sigma}(t)$ and taking into account that $T_{k} \in\left[\mathscr{T}_{1}, \mathscr{T}_{2}\right]$, it follows from (2) that:

$$
\begin{aligned}
\mathscr{T}_{2} \underline{\xi}_{(j)} & \leq \delta_{k(j)}(t) \leq \mathscr{T}_{2} \bar{\xi}_{(j)}, \quad j=1, \cdots, N . \\
\underline{\xi}_{(j)} & \leq \dot{\delta}_{k(j)}(t) \leq \bar{\xi}_{(j)},
\end{aligned}
$$

From the bounds defined in (8), it follows that

$$
\begin{aligned}
\sigma\left(t_{k}\right) \in \mathscr{B}_{\sigma} & =\operatorname{Co}\left\{v_{1}, v_{2}, \ldots, v_{2^{N}}\right\}, \\
\delta_{k}(t) \in \mathscr{B}_{\delta_{k}} & =\operatorname{Co}\left\{\beta_{1}, \beta_{2}, \ldots, \beta_{2^{N}}\right\}, \\
\dot{\delta}_{k}(t) \in \mathscr{B}_{\dot{\delta}}=\mathscr{B}_{\dot{\sigma}} & =\operatorname{Co}\left\{\eta_{1}, \eta_{2}, \ldots, \eta_{2^{N}}\right\},
\end{aligned}
$$

with $\beta_{j}=\mathscr{T}_{2} \eta_{j}, j=1, \ldots, 2^{N}$. Based on (7), we can therefore rewrite $A(\sigma(t))$ given in (3) as follows:

$$
A(\sigma(t))=\left(A_{0}+\mathbf{A}\left[\left(\sigma\left(t_{k}\right)+\delta_{k}(t)\right) \otimes I\right]\right)=A_{0}+\mathbf{A} \Lambda\left(\sigma\left(t_{k}\right)+\delta_{k}(t)\right) .
$$


As pointed out in [23], at the sampling instant $t_{k}$, the model of the closed-loop system formed by (1) and (5) depends on parameter $\sigma\left(t_{k}\right)$, that can take any value in $\mathscr{B}_{\sigma}$. On the other hand, in the interval $\left(t_{k}, t_{k+1}\right)$, the dynamics depends also on the continuous evolution of $\delta_{k}(t)$, which evolves in the polytope $\mathscr{B}_{\delta_{k}}$. It should be noticed that, depending on the maximal admissible intersampling time $\mathscr{T}_{2}$ and the bounds on the derivative of the plant parameters, $\mathscr{B}_{\delta_{k}}$ is potentially much smaller than $\mathscr{B}_{\sigma}$. This formulation leads to a conservatism reduction when compared to the approach considered in [15], where the sampled-parameter used for control purposes $\left(\sigma\left(t_{k}\right)\right)$ and the continuous-time plant parameter $(\sigma(t))$ are treated as independent variables taking arbitrary values in $\mathscr{B}_{\sigma}$.

On the other hand, it is worth noting that, due to the saturation function in (1), the closed-loop system formed by (1) and (5) is nonlinear. Hence, global stabilization of the origin may not be achievable [26]. In this case, the set of all initial conditions $\left(x(0) \in \mathbb{R}^{n}\right)$ such that the corresponding trajectories of system formed by (1) and (5) converge asymptotically to the origin defines the socalled region of attraction of the origin $\left(\mathscr{R}_{a}\right)[26]$. Since the analytical determination of $\mathscr{R}_{a}$ is in general not possible, a problem of interest consists in ensuring asymptotic stability for a set of admissible initial conditions $\mathscr{R}_{0} \subset \mathscr{R}_{a} \subseteq \mathbb{R}^{n}$, taking into account both the input constraints and that the interval between two successive sampling instants may vary, while it is ensured that $J\left(t_{k}, \infty\right) \leq \gamma_{k}$ for some $\gamma_{k}>0$.

Then, based on this formulation we are concerned by the following problem:

Problem 1. Compute $K_{j}$, for $j=0, \ldots, N$, such that an upper bound $\gamma_{k}$ to the criterion $J\left(t_{k}, \infty\right)$ defined in (4) is minimized and the trajectories of the closed-loop system formed by (1) and (5) converge asymptotically to the origin provided that $x\left(t_{k}\right) \in \mathscr{R}_{0}$.

This problem is the core of the MPC strategy to be proposed. Conditions to provide a solution to it will be given in Section 3 .

Remark 1. Note that the measurement of the time-varying parameters is the central idea of LPV control laws. If the time-varying parameters cannot be measured, the control law cannot depend on them. In this case, we refer to a robust control problem, i.e. a parameter-independent control law that ensures stability and performance for the given range of parameter variation is considered. In our case, this solution would correspond to the following particular case of (5):

$$
u(t)=K_{0} x\left(t_{k}\right), \quad \forall t \in\left[t_{k}, t_{k+1}\right), \quad \forall k \in \mathbb{N},
$$

i.e. the matrices $K_{j}, j=1, \ldots, N$ are set equal to zero.

\subsection{MPC Strategy}

In this work, we follow the MPC approach proposed in [1] and [11], where at each sampling instant $t_{k}$, based on the measurement of the states and the parameters, a state feedback control law is computed in order to minimize an infinite-horizon quadratic criterion as given in (4).

With this aim, from the LPV system (1), the following prediction model is considered:

$$
\dot{x}(t)=A(\sigma(t)) x(t)+\operatorname{Bsat}\left(u\left(t_{k+s} \mid t_{k}\right)\right), \quad t \in\left[t_{k+s}, t_{k+s+1}\right), s \geq 0,
$$

where $u\left(t_{k+s} \mid t_{k}\right)$ denotes the control signal to be applied in interval $\left[t_{k+s}, t_{k+s+1}\right)$ from the control law computed at instant $t=t_{k}$. In this work, we consider an LPV state feedback control law as given in (5), i.e.

$$
u\left(t_{k+s} \mid t_{k}\right)=K_{k}\left(\sigma\left(t_{k+s}\right)\right) x\left(t_{k+s} \mid t_{k}\right), \quad t \in\left[t_{k+s}, t_{k+s+1}\right), \quad s \geq 0,
$$


where $x\left(t_{k+s} \mid t_{k}\right)$ denotes the state $x\left(t_{k+s}\right)$ predicted at time $t_{k}$, respectively, for $s=0,1, \ldots, \infty$. Matrix $K_{k}(\sigma)$ is the solution to an optimization problem, computed at time $t_{k}$, aiming at minimizing the upper bound $\gamma_{k}$ on the quadratic criterion $J\left(t_{k}, \infty\right)$ given in (4) using the prediction model (10). Considering the initial condition $x\left(t_{k}\right)$, the computed control law (11) must ensure the asymptotic convergence of the trajectories to the origin of (1) for any function $\sigma(t), t \in\left[t_{k}, \infty\right)$, satisfying (2). Hence, the optimal control sequence obtained at time $t_{k}$ is, in fact, given by the solution of Problem 1 considering $x\left(t_{k}\right)$ as initial condition. Then, the first element of the control sequence $u\left(t_{k} \mid t_{k}\right)$ is applied to the plant, until a new sample is available. Following a receding horizon policy, at next sampling time, $t_{k+1}$, new measurements $x\left(t_{k+1}\right)$ and $\sigma\left(t_{k+1}\right)$ are obtained and a new parameter-dependent feedback gain $K_{k+1}(\sigma)$ is computed to minimize an upper bound $\gamma_{k+1}$ on $J\left(t_{k+1}, \infty\right)$.

The LPV-MPC sampled-data control strategy can therefore be summarized in the following algorithm.

\section{Algorithm 1.}

Step 1: Set k=0;

Step 2: Measure the states and the parameters at the instant $t_{k}$;

Step 3: Compute $K_{k}(\sigma)=K_{k, 0}+\sum_{j=1}^{N} \sigma_{(j)} K_{k, j}$, i.e. determine matrices $K_{k, j}, j=0, \ldots, N$, such that Problem 1 is solved;

Step 4: For $t \in\left[t_{k}, t_{k+1}\right)$, apply the control signal $u(t)=K_{k}\left(\sigma\left(t_{k}\right)\right) x\left(t_{k}\right)$ to the system;

Step 5: Set $k \leftarrow k+1$ and wait for the next sampling instant, then go to Step 2.

From this algorithm, the dynamics of the closed-loop system can be described by

$$
\dot{x}(t)=A(\sigma(t)) x(t)+B s a t\left(K_{k}\left(\sigma\left(t_{k}\right)\right) x\left(t_{k}\right)\right), \quad \forall t \in\left[t_{k}, t_{k+1}\right), \quad \forall k \in \mathbb{N} .
$$

It should be noticed that $\sigma(t)$ is not considered constant neither over the prediction horizon nor between two successive samples. This is, by the way, a difference of this work with respect to classical discrete-time approaches [8-11, 20]. Prediction model (10) assumes that the system dynamics depends continuously on $\sigma(t)$. On the other hand, the control signal applied between $\left[t_{k}, t_{k+1}\right)$ depends only on the value of $\sigma\left(t_{k}\right)$. As $\sigma(t)$ is assumed to evolve in the set $\mathscr{B}_{\sigma}$, the computation of the control signal at time $t_{k}$ and the associated upper bound $\gamma_{k}$ for $J\left(t_{k}, \infty\right)$ must implicitly consider all the possible trajectories for $\sigma(t)$ from $t_{k}$ to $\infty$ that satisfy the bounds given in (2). This will be ensured by convexity properties, as it will be seen in Theorem 2 in the next section.

\section{Guaranteed Cost Sampled-data LPV Control under Saturating Inputs}

In this section, we propose a solution to Problem 1, which is required in Step 3 of Algorithm 1. With this aim, we firstly introduce some preliminary results regarding saturation operators and parameter-dependent quadratic functions. Then, we present the looped-functional approach, which will be applied to obtain LMI based conditions to design the LPV control law (5) in order to solve Problem 1.

In particular, we extend the original results proposed in [21] and [22] to consider parameterdependent functionals. Furthermore, to cope with control saturation, a similar approach to [27] is considered. 


\subsection{Saturation Handling}

From the saturation function definition and (5), for $t \in\left[t_{k}, t_{k+1}\right)$, the following vector-valued deadzone function can be defined:

$$
\psi_{k}=\psi\left(u\left(t_{k}\right)\right)=\operatorname{sat}\left(K\left(\sigma\left(t_{k}\right)\right) x\left(t_{k}\right)\right)-K\left(\sigma\left(t_{k}\right)\right) x\left(t_{k}\right) .
$$

Regarding the nonlinear function $\psi_{k}$, a parameter-dependent version of the generalized sector condition proposed in [26] is stated in the following Lemma.

Lemma 1. [28] Consider the deadzone nonlinearity defined in (13) and a matrix $S\left(\sigma\left(t_{k}\right)\right) \in \mathbb{R}^{m \times n}$. If $x\left(t_{k}\right)$ is such that

$$
\left|\left(K\left(\sigma\left(t_{k}\right)\right)-S\left(\sigma\left(t_{k}\right)\right)\right)_{(i)} x\left(t_{k}\right)\right| \leq \bar{u}_{(i)}, \text { for } i=1, \ldots, m,
$$

then the following inequality is satisfied for any diagonal positive definite matrix $U\left(\sigma\left(t_{k}\right)\right) \in \mathbb{R}^{m \times m}$ :

$$
\psi_{k}^{\prime} U\left(\sigma\left(t_{k}\right)\right)\left(\psi_{k}+S\left(\sigma\left(t_{k}\right)\right) x\left(t_{k}\right)\right) \leq 0 .
$$

In what follows, as $K\left(\sigma\left(t_{k}\right)\right)$ is affine on $\sigma\left(t_{k}\right)$, we will consider that $S\left(\sigma\left(t_{k}\right)\right)$ is also affine on $\sigma\left(t_{k}\right)$, that is:

$$
S\left(\sigma\left(t_{k}\right)\right)=S_{0}+\mathbf{S} \Lambda\left(\sigma\left(t_{k}\right)\right)
$$

where $\mathbf{S}=\left[S_{1} \ldots S_{N}\right]$.

\subsection{Parameter-Dependent Quadratic Function}

Consider a parameter-dependent quadratic function (PDQF) $V: \mathbb{R}^{n} \times \mathscr{B}_{\sigma} \rightarrow \mathbb{R}^{+}$given by

$$
V(x, \sigma)=x^{\prime} P(\sigma) x,
$$

with $P(\sigma) \in \mathbb{S}^{n}$ and $P(\sigma)>0$, for all $\sigma \in \mathscr{B}_{\sigma}$. Assuming again an affine dependence on the parameter, it follows that

$$
P(\sigma)=P_{0}+\mathbf{P}[\boldsymbol{\sigma} \otimes I]=P_{0}+\mathbf{P}\left(\sum_{f=1}^{2^{N}} \lambda_{f} v_{f} \otimes I\right)=\sum_{f=1}^{2^{N}} \lambda_{f}\left(P_{0}+\mathbf{P} \Lambda\left(v_{f}\right)\right)
$$

where $\mathbf{P}=\left[P_{1} \ldots P_{N}\right], \lambda_{f} \in[0,1]$, with $\sum_{f=1}^{2^{N}} \lambda_{f}=1$, and $v_{f}$ given in (9).

For $c>0$, the level set associated to the PDQF (16) is given by

$$
\mathscr{L}_{V}(c)=\left\{x \in \mathbb{R}^{n} ; V(x, \sigma) \leq c, \forall \sigma \in \mathscr{B}_{\sigma}\right\}=\bigcap_{\sigma \in \mathscr{B}_{\sigma}} \mathscr{E}(P(\sigma), c),
$$

where $\mathscr{E}(P(\sigma), c)=\left\{x \in \mathbb{R}^{n} ; x^{\prime} P(\sigma) x \leq c\right\}$.

Inspired by the results of [29], the following lemma can be stated.

Lemma 2. $x \in \mathscr{L}_{V}(c)$ if and only if $x \in \bigcap_{f \in\left\{1, \ldots, 2^{N}\right\}} \mathscr{E}\left(P_{0}+\boldsymbol{P} \Lambda\left(v_{f}\right), c\right)$. 
Proof. The sufficiency holds directly from the fact that $\bigcap_{\sigma \in \mathscr{B}_{\sigma}} \mathscr{E}(P(\sigma), c) \subseteq \bigcap_{f \in\left\{1, \ldots, 2^{N}\right\}} \mathscr{E}\left(P_{0}+\right.$ $\left.\mathbf{P} \Lambda\left(v_{f}\right), c\right)$. To demonstrate the necessity, note that if $x \in \bigcap_{f \in\left\{1, \ldots, 2^{N}\right\}} \mathscr{E}\left(P_{0}+\mathbf{P} \Lambda\left(v_{f}\right), c\right)$ then $x^{\prime}\left(P_{0}+\right.$ $\left.\mathbf{P} \Lambda\left(v_{f}\right)\right) x \leq c$, for all $f=1, \ldots, 2^{N}$. Since $\sigma \in \mathscr{B}_{\sigma}$, it follows that $\sigma=\sum_{f=1}^{2^{N}} \lambda_{f} v_{f}$, with $\sum_{f=1}^{2^{N}} \lambda_{f}=1$ and $\lambda_{f} \in[0,1]$. Hence from (17), we obtain:

$$
x^{\prime}\left[\sum_{f=1}^{2^{N}} \lambda_{f}\left(P_{0}+\mathbf{P} \Lambda\left(v_{f}\right)\right)\right] x=x^{\prime}\left[P_{0}+\mathbf{P}\left(\sum_{f=1}^{2^{N}}\left[\lambda_{f} v_{f} \otimes I\right]\right)\right] x=x^{\prime}\left[P_{0}+\mathbf{P}[\boldsymbol{\sigma} \otimes I]\right] x=x^{\prime} P(\sigma) x \leq c,
$$

that is, $x \in \mathscr{L}_{V}(c)$.

Defining now $\Delta V(k)=x^{\prime}\left(t_{k+1}\right) P\left(\sigma\left(t_{k+1}\right)\right) x\left(t_{k+1}\right)-x^{\prime}\left(t_{k}\right) P\left(\sigma\left(t_{k}\right)\right) x\left(t_{k}\right)$, the following lemma can be stated.

Lemma 3. Consider $V: \mathbb{R}^{n} \times \mathscr{B}_{\sigma} \rightarrow \mathbb{R}^{+}$as defined in (16) and a domain $\mathscr{D} \subset \mathbb{R}^{n}$ containing the origin in its interior. If

$$
\Delta V(k)<-\mu\left\|x\left(t_{k}\right)\right\|^{2}, \quad \forall x\left(t_{k}\right) \in \mathscr{D} \backslash\{0\}, \quad \forall k \in \mathbb{N},
$$

along the trajectories of system formed by (1) and (5) with $\mu$ being a positive scalar, then, for any initial condition $x\left(t_{0}\right) \in \mathscr{L}_{V}(c) \subseteq \mathscr{D}$, it follows that:

(i) $\mathscr{L}_{V}(c)$ is an invariant and contractive set with respect to the trajectories of system formed by (1) and (5) at the sampling instants.

(ii) $x\left(t_{k}\right)$ converges to zero as $k \rightarrow \infty$.

Proof. Suppose that $x\left(t_{0}\right)$ belongs to the boundary of $\mathscr{L}_{V}\left(c_{0}\right)$, i.e.

$$
x^{\prime}\left(t_{0}\right) P\left(\sigma\left(t_{0}\right)\right) x\left(t_{0}\right)=c_{0},
$$

with $c_{0} \leq c$. Since $x\left(t_{0}\right) \in \partial \mathscr{L}_{V}\left(c_{0}\right) \subseteq \mathscr{L}_{V}(c) \subseteq \mathscr{D}$, it follows from (19) that

$$
x\left(t_{1}\right) \in \partial \mathscr{L}_{V}\left(c_{1}\right) \subset \mathscr{L}_{V}\left(c_{0}\right) \text {, with } c_{1}<c_{0} .
$$

Repeating this reasoning for $k=1, \ldots, \infty$, we conclude the proof of item $(i)$. Since $\mathscr{L}_{V}(c)$ is a compact set, item (ii) directly follows.

\subsection{The Looped-Functional Approach}

Following the notation in [22], i.e. considering $x_{k}(\tau)=x\left(t_{k}+\tau\right)$ and $\sigma_{k}(\tau)=\sigma\left(t_{k}+\tau\right)$, for $\tau \in\left[0, T_{k}\right)$ and for $k \in \mathbb{N}$, the dynamics of the closed-loop system between two successive sampling instants is given by:

$$
\dot{x}_{k}(\tau)=A\left(\sigma_{k}(\tau)\right) x_{k}(\tau)+B K\left(\sigma_{k}(0)\right) x_{k}(0)+B \psi_{k} .
$$

Considering (20), the following theorem is the basis for the so-called looped-functional approach $[21,22]$ to ensure the asymptotic stability of sampled-data LPV systems under saturating controls, while providing a bound on the cost function $J\left(t_{k}, \infty\right)$ defined in (4). It can be seen as a parameterdependent version of Theorem 1 proposed in [27], applied to the stability and guaranteed cost analysis of LPV systems. 
Theorem 1. Consider a parameter-dependent function (PDF) $V: \mathbb{R}^{n} \times \mathbb{R}^{N} \rightarrow \mathbb{R}^{+}$that satisfies

$$
\mu_{1}\|x\|^{p} \leq V(x, \sigma) \leq \mu_{2}\|x\|^{p},
$$

with $p>0,0<\mu_{1} \leq \mu_{2}$ and a parameter-dependent looped-functional ${ }^{1}(P D L F) \mathscr{V}_{0}:\left[0, \mathscr{T}_{2}\right] \times \mathbb{K}_{\left[\mathscr{T}_{1}, \mathscr{T}_{2}\right]} \times$ $\mathbb{K}_{\left[\mathscr{T}_{1}, \mathscr{T}_{2}\right]}^{N} \times\left[\mathscr{T}_{1}, \mathscr{T}_{2}\right] \rightarrow \mathbb{R}$ verifying

$$
\mathscr{V}_{0}\left(0, x_{k}, \sigma_{k}, T_{k}\right)=\mathscr{V}_{0}\left(T_{k}, x_{k}, \sigma_{k}, T_{k}\right) .
$$

Define the functional $\mathscr{W}\left(\tau, x_{k}, \sigma_{k}, T_{k}\right)=V\left(x_{k}(\tau), \sigma_{k}(\tau)\right)+\mathscr{V}_{0}\left(\tau, x_{k}, \sigma_{k}, T_{k}\right)$ and let $\dot{\mathscr{W}}\left(\tau, x_{k}, \sigma_{k}, T_{k}\right)$ be the derivative of $\mathscr{W}\left(\tau, x_{k}, \sigma_{k}, T_{k}\right)$ with respect to $\tau$ along the trajectories of system (20). If there exist positive scalars $\mu$ and $\gamma_{k}$, a matrix $S\left(\sigma_{k}(0)\right) \in \mathbb{R}^{m \times n}$ and a diagonal positive definite matrix $U\left(\sigma_{k}(0)\right) \in \mathbb{S}^{m}$ such that the following inequalities are satisfied ${ }^{2}$

$$
\begin{array}{r}
\left|\left(K\left(\sigma_{k}(0)\right)-S\left(\sigma_{k}(0)\right)\right)_{(i)} x_{k}(0)\right|^{2} \leq \bar{u}_{(i)}^{2} \frac{V\left(x_{k}(0), \sigma_{k}(0)\right)}{\gamma_{k}}, \quad \forall i=1, \ldots, m, \\
\dot{\mathscr{W}}+\left\|x_{k}(\tau)\right\|_{Z}^{2}+\left\|\operatorname{sat}\left(u_{k}(\tau)\right)\right\|_{H}^{2}-2 \psi_{k}^{\prime} U\left(\sigma_{k}(0)\right)\left(\psi_{k}+S\left(\sigma_{k}(0)\right) x_{k}(0)\right)<-\mu\left\|\left[\begin{array}{l}
x_{k}(\tau) \\
x_{k}(0)
\end{array}\right]\right\|^{p}, \\
\quad \text { for }\left[\begin{array}{l}
x_{k}(\tau) \\
x_{k}(0)
\end{array}\right] \neq 0,
\end{array}
$$

for all $\tau \in\left[0, T_{k}\right), \forall T_{k} \in\left[\mathscr{T}_{1}, \mathscr{T}_{2}\right]$ and $\forall \sigma_{k}(\tau) \in \mathscr{B}_{\sigma}, \dot{\sigma}_{k}(\tau) \in \mathscr{B}_{\dot{\delta}}$, then, provided that $x\left(t_{k}\right)=x_{k}(0) \in$ $\mathscr{L}_{V}\left(\gamma_{k}\right)=\left\{x \in \mathbb{R}^{n} ; V(x, \sigma) \leq \gamma_{k}, \forall \sigma \in \mathscr{B}_{\sigma}\right\}$ it follows that:

(i) the corresponding trajectories of the closed-loop system formed by (1) and (5), with $\sigma$ satisfying (2) and intersampling time $T_{k} \in\left[\mathscr{T}_{1}, \mathscr{T}_{2}\right]$, converge asymptotically to the origin;

(ii) $J\left(t_{k}, \infty\right)<\gamma_{k}$.

Proof. Suppose $x\left(t_{k}\right)=x_{k}(0) \in \mathscr{L}_{V}\left(\gamma_{k}\right)$. Thus $V\left(x_{k}(0), \sigma_{k}(0)\right) \leq \gamma_{k}$ and, if (23) is satisfied, it follows that

$$
\left|\left(K\left(\sigma_{k}(0)\right)_{(i)}-S\left(\sigma_{k}(0)\right)_{(i)}\right) x_{k}(0)\right| \leq \bar{u}_{(i)},
$$

for $i=1, \ldots, m$. From Lemma 1 , this implies that

$$
\psi_{k}^{\prime} U\left(\sigma_{k}(0)\right)\left(\psi_{k}+S\left(\sigma_{k}(0)\right) x_{k}(0)\right) \leq 0
$$

Taking (25) into account and integrating (24) over any sampling interval gives that

$$
\begin{aligned}
\int_{0}^{T_{k}}\left\{\dot{\mathscr{W}}+\left\|x_{k}(\tau)\right\|_{Z}^{2}+\left\|\operatorname{sat}\left(u_{k}(\tau)\right)\right\|_{H}^{2}\right\} d \tau< \\
\quad 2 \int_{0}^{T_{k}} \psi_{k}^{\prime} U\left(\sigma_{k}(0)\right)\left(\psi_{k}+S\left(\sigma_{k}(0)\right)\right) x_{k}(0) d \tau-\mu \int_{0}^{T_{k}}\left\|\left[\begin{array}{l}
x_{k}(\tau) \\
x_{k}(0)
\end{array}\right]\right\|^{p} d \tau<-\mu \int_{0}^{T_{k}}\left\|\left[\begin{array}{l}
x_{k}(\tau) \\
x_{k}(0)
\end{array}\right]\right\|^{p} d \tau .
\end{aligned}
$$

\footnotetext{
${ }^{1}$ See definition of looped-functionals in [21] for more details.

${ }^{2}$ For simplicity, the argument of $\mathscr{\mathscr { W }}\left(\tau, x_{k}, \sigma_{k}, T_{k}\right)$ will be omitted.
} 
Since from (22) we have $\mathscr{V}_{0}\left(0, x_{k}, \sigma_{k}, T_{k}\right)=\mathscr{V}_{0}\left(T_{k}, x_{k}, \sigma_{k}, T_{k}\right)$, it follows from (26) that

$$
\begin{aligned}
V\left(x_{k}\left(T_{k}\right), \sigma_{k}\left(T_{k}\right)\right)-V\left(x_{k}(0), \sigma_{k}(0)\right)+\int_{0}^{T_{k}}\left(\left\|x_{k}(\tau)\right\|_{Z}^{2}+\left\|\operatorname{sat}\left(u_{k}(\tau)\right)\right\|_{H}^{2}\right) d \tau< \\
\quad-\int_{0}^{T_{k}} \mu\left\|\left[\begin{array}{l}
x_{k}(\tau) \\
x_{k}(0)
\end{array}\right]\right\|^{p} d \tau \leq-\int_{0}^{T_{k}} \mu\left\|x_{k}(0)\right\|^{p} d \tau \leq-T_{k} \mu\left\|x_{k}(0)\right\|^{p} .
\end{aligned}
$$

Recalling that by continuity $x_{k}\left(T_{k}\right)=x_{k+1}(0)$ and $\sigma_{k}\left(T_{k}\right)=\sigma_{k+1}(0)$, from (27) we have that

$$
\Delta V(k)<-T_{k} \mu\left\|x_{k}(0)\right\|^{p} \leq-\mathscr{T}_{1} \mu\left\|x_{k}(0)\right\|^{p} .
$$

As a consequence of $(28)$, we can conclude that $\lim _{k \rightarrow \infty} x_{k}(0)=0$. Moreover, since the open-loop system is linear and $\sigma(t)$ is bounded in $\mathscr{B}_{\sigma}$, it is possible to conclude (see details in [23]) that there exists a positive scalar $\mu_{m}$ such that $\left\|x_{k}(\tau)\right\| \leq \mu_{m}\left\|x_{k}(0)\right\|, \forall k \geq 0$. Hence, if $\lim _{k \rightarrow \infty} x_{k}(0)=0$, it follows that $x_{k}(\tau)=x\left(t_{k}+\tau\right) \rightarrow 0$ as $k \rightarrow \infty$, for any $\tau \in\left[0, T_{k}\right)$. This reasoning concludes the proof of item $(i)$.

Now we prove item $(i i)$. Noting that

$$
\int_{0}^{T_{k}}\left(\left\|x_{k}(\tau)\right\|_{Z}^{2}+\left\|\operatorname{sat}\left(u_{k}(\tau)\right)\right\|_{H}^{2}\right) d \tau=\int_{t_{k}}^{t_{k+1}}\left(\|x(t)\|_{Z}^{2}+\|\operatorname{sat}(u(t))\|_{H}^{2}\right) d t
$$

from (26) one gets:

$$
\begin{aligned}
& \sum_{a=k}^{k+s} \int_{0}^{T_{a}}\left\{\dot{\mathscr{W}}+\left\|x_{a}(\tau)\right\|_{Z}^{2}+\left\|\operatorname{sat}\left(u_{a}(\tau)\right)\right\|_{H}^{2}\right\} d \tau=V\left(x_{k+s+1}(0), \sigma_{k+s+1}(0)\right)-V\left(x_{k}(0), \sigma_{k}(0)\right) \\
&+J\left(t_{k}, t_{k+s+1}\right)<0
\end{aligned}
$$

and, consequently,

$$
J\left(t_{k}, t_{k+s+1}\right)<-V\left(x_{k+s+1}(0), \sigma_{k+s+1}(0)\right)+V\left(x_{k}(0), \sigma_{k}(0)\right) .
$$

Now considering $s \rightarrow \infty$ in $(29)$ and the fact that $\lim _{s \rightarrow \infty} x_{k+s+1}(0)=0$, we conclude that

$$
J\left(t_{k}, \infty\right)<V\left(x_{k}(0), \sigma_{k}(0)\right) \leq \gamma_{k} .
$$

\subsection{Stabilization Conditions}

From Theorem 1, we derive now conditions to compute a parameter-dependent state-feedback gain matrix that solves Problem 1. With this aim, we consider the PDQF $V(x, \sigma)$ defined in (16) and a PDLF candidate $\mathscr{V}_{0}\left(\tau, x_{k}, \sigma_{k}, T_{k}\right)$ defined as follows:

$$
\begin{aligned}
\mathscr{V}_{0}\left(\tau, x_{k}, \sigma_{k}, T_{k}\right)=\left(T_{k}-\tau\right)\left\{\left(x_{k}(\tau)-x_{k}(0)\right)^{\prime}\left[2 G\left(\sigma_{k}(\tau)\right) x_{k}(0)+F\left(\sigma_{k}(\tau)\right)\left(x_{k}(\tau)-x_{k}(0)\right)\right]\right. \\
\left.+\tau\left[\begin{array}{c}
x_{k}(0) \\
\psi_{k}
\end{array}\right]^{\prime} X\left(\sigma_{k}(0)\right)\left[\begin{array}{c}
x_{k}(0) \\
\psi_{k}
\end{array}\right]+\int_{0}^{\tau} \dot{x}_{k}^{\prime}(\theta) R \dot{x}_{k}(\theta) d \theta\right\},
\end{aligned}
$$


where $X\left(\sigma_{k}(0)\right) \in \mathbb{S}^{n+m}, F\left(\sigma_{k}(\tau)\right) \in \mathbb{S}^{n}, G\left(\sigma_{k}(\tau)\right) \in \mathbb{R}^{n \times n}$ and $R \in \mathbb{S}^{n}$, with $R=R^{\prime}>0$. Moreover, we assume that:

$$
\begin{aligned}
& F\left(\sigma_{k}(\tau)\right)=F_{0}+\mathbf{F}\left[\sigma_{k}(\tau) \otimes I\right]=F_{0}+\mathbf{F} \Lambda\left(\sigma_{k}(\tau)\right), \\
& G\left(\sigma_{k}(\tau)\right)=G_{0}+\mathbf{G}\left[\sigma_{k}(\tau) \otimes I\right]=G_{0}+\mathbf{G} \Lambda\left(\sigma_{k}(\tau)\right),
\end{aligned}
$$

with the matrices $\mathbf{F}=\left[\begin{array}{llll}F_{1} & \ldots & F_{N}\end{array}\right]$ and $\mathbf{G}=\left[\begin{array}{lll}G_{1} & \ldots & G_{N}\end{array}\right]$ of appropriate dimensions. Notice that $\mathscr{V}_{0}\left(\tau, x_{k}, \sigma_{k}, T_{k}\right)$ satisfies $(22)$.

Next, based on the PDLF $\mathscr{V}_{0}\left(\tau, x_{k}, \sigma_{k}, T_{k}\right)$ given in (30) and the PDQF $V(x, \sigma)$ defined in (16), constructive conditions to verify (23)-(24) of Theorem 1 are proposed.

Theorem 2. If there exist symmetric matrices $\tilde{P}_{j}, \tilde{F}_{j} \in \mathbb{S}^{n}, \tilde{X}_{j} \in \mathbb{S}^{n+m}$, matrices $\tilde{G}_{j}, \tilde{Y} \in \mathbb{R}^{n \times n}$, $\tilde{Q}_{j} \in \mathbb{R}^{3 n+m \times n}, \tilde{K}_{j}, \tilde{S}_{j} \in \mathbb{R}^{m \times n}$, diagonal matrices $\tilde{U}_{j} \in \mathbb{S}^{m}$, for $j=0,1, \cdots, N$, a positive definite matrix $\tilde{R} \in \mathbb{S}^{n}$ and positive scalars $\mathcal{\varepsilon}$ and $\gamma_{k}$ satisfying the following matrix inequalities:

$$
\begin{aligned}
& \Psi_{1}\left(v, \beta, \eta, \mathscr{T}_{r}\right)<0, \\
& \Psi_{2}\left(v, \beta, \eta, \mathscr{T}_{r}\right)<0, \\
& {\left[\begin{array}{cc}
\tilde{P}_{0}+\tilde{\boldsymbol{P}} \Lambda(v) & \left(\tilde{K}_{0}+\tilde{\boldsymbol{K}} \Lambda(v)\right)_{(i)}^{\prime}-\left(\tilde{S}_{0}+\tilde{\boldsymbol{S}} \Lambda(v)\right)_{(i)}^{\prime} \\
* & \bar{u}_{(i)}^{2}
\end{array}\right]>0,} \\
& \tilde{U}_{0}+\tilde{\boldsymbol{U}} \Lambda(v)>0, \\
& \tilde{P}_{0}+\tilde{\boldsymbol{P}} \Lambda(v+\beta)>0,
\end{aligned}
$$

for all $(v, \beta, \eta) \in \operatorname{Ver}\left(\mathscr{B}_{\sigma}\right) \times \operatorname{Ver}\left(\mathscr{B}_{\delta_{k}}\right) \times \operatorname{Ver}\left(\mathscr{B}_{\dot{\delta}}\right), \forall i=1, \ldots, m$ and $\forall r=1,2$, with

$\Psi_{1}\left(v, \beta, \eta, \mathscr{T}_{r}\right)=$

$$
\left[\begin{array}{ccc}
\tilde{\Pi}_{1}(v, \beta, \eta)+\mathscr{T}_{r}\left(\tilde{\Pi}_{2}(v, \beta, \eta)+\tilde{\Pi}_{3}(v)\right) & M_{1}^{\prime} \tilde{Y}^{\prime} \bar{Z}^{\prime} & M_{2}^{\prime}\left(\tilde{K}_{0}+\tilde{\boldsymbol{K}} \Lambda(v)\right)^{\prime}+M_{4}^{\prime}\left(\tilde{U}_{0}+\tilde{\boldsymbol{U}} \Lambda(v)\right) \\
* & -\gamma_{k} I & 0 \\
* & * & -\gamma_{k} H^{-1}
\end{array}\right],
$$

$\Psi_{2}\left(v, \beta, \eta, \mathscr{T}_{r}\right)=$

$$
\left[\begin{array}{cccc}
\tilde{\Pi}_{1}(v, \beta, \eta)-\mathscr{T}_{r} \tilde{\Pi}_{3}(v) & \mathscr{T}_{r}\left(\tilde{Q}_{0}+\tilde{\boldsymbol{Q}} \Lambda(v+\beta)\right) & M_{1}^{\prime} \tilde{Y}^{\prime} \bar{Z}^{\prime} & M_{2}^{\prime}\left(\tilde{K}_{0}+\tilde{\boldsymbol{K}} \Lambda(v)\right)^{\prime}+M_{4}^{\prime}\left(\tilde{U}_{0}+\tilde{\boldsymbol{U}} \Lambda(v)\right) \\
* & -\mathscr{T}_{r} \tilde{R} & 0 & 0 \\
* & * & -\gamma_{k} I & 0 \\
* & * & * & -\gamma_{k} H^{-1}
\end{array}\right],
$$

where

$$
\begin{aligned}
\tilde{\Pi}_{1}(v, \beta, \eta)= & H e\left\{M_{1}^{\prime}\left(\tilde{P}_{0}+\tilde{\boldsymbol{P}} \Lambda(v+\beta)\right) M_{3}-M_{12}^{\prime}\left(\tilde{G}_{0}+\tilde{\boldsymbol{G}} \Lambda(v+\beta)\right) M_{2}-\left(\tilde{Q}_{0}+\tilde{\boldsymbol{Q}} \Lambda(v+\beta)\right) M_{12}\right. \\
& +\left(\varepsilon M_{1}^{\prime}+M_{3}^{\prime}\right)\left(-\tilde{Y} M_{3}+\left(A_{0}+\boldsymbol{A} \Lambda(v+\beta)\right) \tilde{Y} M_{1}+B\left(\tilde{K}_{0}+\tilde{\boldsymbol{K}} \Lambda(v)\right) M_{2}\right. \\
& \left.\left.+B\left(\tilde{U}_{0}+\tilde{\boldsymbol{U}} \Lambda(v)\right) M_{4}\right)-M_{4}^{\prime}\left(\tilde{S}_{0}+\tilde{\boldsymbol{S}} \Lambda(v)\right) M_{2}\right\}-M_{12}^{\prime}\left(\tilde{F}_{0}+\tilde{\boldsymbol{F}} \Lambda(v+\beta)\right) M_{12} \\
& +M_{1}^{\prime} \tilde{\boldsymbol{P}} \Lambda(\eta) M_{1}-2 M_{4}^{\prime}\left(\tilde{U}_{0}+\tilde{\boldsymbol{U}} \Lambda(v)\right) M_{4}, \\
\tilde{\Pi}_{2}(v, \beta, \eta)= & \operatorname{He}\left\{M_{3}^{\prime}\left(\left(\tilde{\boldsymbol{G}}_{0}+\tilde{\boldsymbol{G}} \Lambda(v+\beta)\right) M_{2}+\left(\tilde{F}_{0}+\tilde{\boldsymbol{F}} \Lambda(v+\beta)\right) M_{12}\right)+M_{12}^{\prime} \tilde{\boldsymbol{G}} \Lambda(\eta) M_{2}\right\}+M_{3}^{\prime} \tilde{R} M_{3} \\
& +M_{12}^{\prime} \tilde{\boldsymbol{F}} \Lambda(\eta) M_{12}, \\
\tilde{\Pi}_{3}(v)= & M_{24}^{\prime}\left(\tilde{X}_{0}+\tilde{\boldsymbol{X}} \Lambda(v)\right) M_{24},
\end{aligned}
$$




$$
\begin{aligned}
& M_{1}=\left[\begin{array}{llll}
I & 0 & 0 & 0
\end{array}\right], \quad M_{2}=\left[\begin{array}{llll}
0 & I & 0 & 0
\end{array}\right], \quad M_{12}=M_{1}-M_{2} \text {, } \\
& M_{3}=\left[\begin{array}{llll}
0 & 0 & I & 0
\end{array}\right], \quad M_{4}=\left[\begin{array}{llll}
0 & 0 & 0 & I
\end{array}\right], \quad M_{24}=\left[\begin{array}{ll}
M_{2}^{\prime} & M_{4}^{\prime}
\end{array}\right]^{\prime} \text {, }
\end{aligned}
$$

then the sampled-data control law (5) with the gain matrix

$$
K\left(\sigma\left(t_{k}\right)\right)=K_{0}+\boldsymbol{K}\left[\sigma\left(t_{k}\right) \otimes I\right],
$$

where $K_{j}=\tilde{K}_{j} \tilde{Y}^{-1}, j=0,1, \ldots, N$ is such that $\forall x\left(t_{k}\right) \in \mathscr{L}_{V}\left(\gamma_{k}\right)$, where $\mathscr{L}_{V}\left(\gamma_{k}\right)$ is defined as in (18) with $P(\sigma(t))$ defined by matrices $P_{j}=\gamma_{k}\left(\tilde{Y}^{\prime}\right)^{-1} \tilde{P}_{j} \tilde{Y}^{-1}$, for $j=0,1, \ldots, N$, ensures that:

(i) the corresponding trajectories of the closed-loop system formed by (1) and (5), with $\sigma$ satisfying (2) and intersampling time $T_{k} \in\left[\mathscr{T}_{1}, \mathscr{T}_{2}\right]$, converge asymptotically to the origin;

(ii) $J\left(t_{k}, \infty\right)<\gamma_{k}$.

Proof. This proof is based on the results of Theorem 1, considering the PDQF $V(x, \sigma)$ and the PDLF $\mathscr{V}_{0}\left(\tau, x_{k}, \sigma_{k}, T_{k}\right)$ defined in (16) and (30), that satisfy (21) and (22), respectively. Differentiating $\mathscr{W}\left(\tau, x_{k}, \sigma_{k}, T_{k}\right)=V\left(x_{k}(\tau), \sigma_{k}(\tau)\right)+\mathscr{V}_{0}\left(\tau, x_{k}, \sigma_{k}, T_{k}\right)$ with respect to $\tau$ one obtains

$$
\begin{aligned}
\dot{\mathscr{W}}= & 2 \dot{x}_{k}^{\prime}(\tau) P\left(\sigma_{k}(\tau)\right) x_{k}(\tau)+x_{k}^{\prime}(\tau) \dot{P}\left(\sigma_{k}(\tau)\right) x_{k}(\tau)-\left(x_{k}(\tau)-x_{k}(0)\right)^{\prime}\left[F\left(\sigma_{k}(\tau)\right)\left(x_{k}(\tau)-x_{k}(0)\right)\right. \\
& \left.+2 G\left(\sigma_{k}(\tau)\right) x_{k}(0)\right]+\left(T_{k}-\tau\right)\left[2 \dot{x}_{k}^{\prime}(\tau) F\left(\sigma_{k}(\tau)\right)\left(x_{k}(\tau)-x_{k}(0)\right)+2 \dot{x}_{k}^{\prime}(\tau) G\left(\sigma_{k}(\tau)\right) x_{k}(0)\right. \\
& \left.+\left(x_{k}(\tau)-x_{k}(0)\right)^{\prime} \dot{F}\left(\sigma_{k}(\tau)\right)\left(x_{k}(\tau)-x_{k}(0)\right)+2\left(x_{k}(\tau)-x_{k}(0)\right)^{\prime} \dot{G}\left(\sigma_{k}(\tau)\right) x_{k}(0)\right] \\
& +\left(T_{k}-2 \tau\right)\left[\begin{array}{c}
x_{k}(0) \\
\psi_{k}
\end{array}\right]^{\prime} X\left(\sigma_{k}(0)\right)\left[\begin{array}{c}
x_{k}(0) \\
\psi_{k}
\end{array}\right]+\left(T_{k}-\tau\right) \dot{x}_{k}^{\prime}(\tau) R \dot{x}_{k}(\tau)-\int_{0}^{\tau} \dot{x}_{k}^{\prime}(\theta) R \dot{x}_{k}(\theta) d \theta,
\end{aligned}
$$

where

$$
\dot{P}\left(\sigma_{k}(\tau)\right)=\mathbf{P} \Lambda\left(\dot{\delta}_{k}(\tau)\right), \quad \dot{F}\left(\sigma_{k}(\tau)\right)=\mathbf{F} \Lambda\left(\dot{\delta}_{k}(\tau)\right), \quad \dot{G}\left(\sigma_{k}(\tau)\right)=\mathbf{G} \Lambda\left(\dot{\delta}_{k}(\tau)\right)
$$

Consider now a matrix $Q\left(\sigma_{k}(\tau)\right)=Q_{0}+\mathbf{Q} \Lambda\left(\sigma_{k}(\tau)\right)$, with $\mathbf{Q}=\left[\begin{array}{lll}Q_{1} & Q_{2} & \ldots\end{array} Q_{N}\right]$ and $Q_{j} \in \mathbb{R}^{(3 n+m) \times n}$ for $j=0, \ldots, N$, and define

$$
\chi_{k}(\tau)=\left[\begin{array}{llll}
x_{k}^{\prime}(\tau) & x_{k}^{\prime}(0) & \dot{x}_{k}^{\prime}(\tau) & \psi_{k}^{\prime}
\end{array}\right]^{\prime} .
$$

Next, we consider an upper bound for the integral term that appears in (39). Since $R>0$, it follows that $\left(\dot{x}_{k}(\theta)-R^{-1} Q^{\prime}\left(\sigma_{k}(\tau)\right) \chi_{k}(\tau)\right)^{\prime} R\left(\dot{x}_{k}(\theta)-R^{-1} Q^{\prime}\left(\sigma_{k}(\tau)\right) \chi_{k}(\tau)\right) \geq 0$, for $\theta \in[0, \tau]$. Integrating this inequality over the interval $[0, \tau]$ leads to $[30]$ :

$$
\int_{0}^{\tau} \dot{x}_{k}^{\prime}(\theta) R \dot{x}_{k}(\theta) d \theta \geq 2 \chi_{k}^{\prime}(\tau) Q\left(\sigma_{k}(\tau)\right)\left(x_{k}(\tau)-x_{k}(0)\right)-\tau \chi_{k}^{\prime}(\tau) Q\left(\sigma_{k}(\tau)\right) R^{-1} Q^{\prime}\left(\sigma_{k}(\tau)\right) \chi_{k}(\tau)
$$

Considering now (39) and (40), the auxiliary matrices defined in $(38)$ and that $\sigma_{k}(\tau)=\sigma_{k}(0)+$ $\delta_{k}(\tau)$, one obtains the following:

$$
\begin{aligned}
\dot{\mathscr{W}}- & 2 \psi_{k}^{\prime} U\left(\sigma_{k}(0)\right)\left[\psi_{k}+S\left(\sigma_{k}(0)\right) x_{k}(0)\right]+x_{k}^{\prime}(\tau) Z x_{k}(\tau)+\operatorname{sat}\left(u_{k}(\tau)\right)^{\prime} \operatorname{Hsat}\left(u_{k}(\tau)\right) \leq \\
& \chi_{k}^{\prime}(\tau)\left[\hat{\Pi}_{1}\left(\sigma_{k}(0), \delta_{k}(\tau), \dot{\delta}_{k}(\tau)\right)+\left(T_{k}-\tau\right) \Pi_{2}\left(\sigma_{k}(0), \delta_{k}(\tau), \dot{\delta}_{k}(\tau)\right)+\left(T_{k}-2 \tau\right) \Pi_{3}\left(\sigma_{k}(0)\right)\right. \\
& \left.+\tau\left(Q_{0}+\mathrm{Q} \Lambda\left(\sigma_{k}(0)+\delta_{k}(\tau)\right)\right) R^{-1}\left(Q_{0}+\mathrm{Q} \Lambda\left(\sigma_{k}(0)+\delta_{k}(\tau)\right)\right)^{\prime}\right] \chi_{k}(\tau) \\
& +x_{k}^{\prime}(\tau) Z x_{k}(\tau)+\operatorname{sat}\left(u_{k}(\tau)\right)^{\prime} \operatorname{Hsat}\left(u_{k}(\tau)\right),
\end{aligned}
$$


with

$$
\begin{aligned}
\hat{\Pi}_{1}\left(\sigma_{k}(0), \delta_{k}(\tau), \dot{\delta}_{k}(\tau)\right)= & M_{1}^{\prime} \mathbf{P} \Lambda\left(\dot{\delta}_{k}(\tau)\right) M_{1}-2 M_{4}^{\prime} U\left(\sigma_{k}(0)\right) M_{4}+\operatorname{He}\left\{M_{3}^{\prime}\left(P_{0}+\mathbf{P} \Lambda\left(\sigma_{k}(0)+\delta_{k}(\tau)\right)\right) M_{1}\right. \\
& -\left(Q_{0}+\mathbf{Q} \Lambda\left(\sigma_{k}(0)+\delta_{k}(\tau)\right)\right) M_{12}-M_{12}^{\prime}\left(G_{0}+\mathbf{G} \Lambda\left(\sigma_{k}(0)+\delta_{k}(\tau)\right)\right) M_{2} \\
& \left.-M_{4}^{\prime} U\left(\sigma_{k}(0)\right)\left(S_{0}+\mathbf{S} \Lambda\left(\sigma_{k}(0)\right)\right) M_{2}\right\}-M_{12}^{\prime}\left(F_{0}+\mathbf{F} \Lambda\left(\sigma_{k}(0)+\delta_{k}(\tau)\right)\right) M_{12}, \\
\Pi_{2}\left(\sigma_{k}(0), \delta_{k}(\tau), \dot{\delta}_{k}(\tau)\right)= & M_{3}^{\prime} R M_{3}+\operatorname{He}\left\{M_{3}^{\prime}\left(F_{0}+\mathbf{F} \Lambda\left(\sigma_{k}(0)+\delta_{k}(\tau)\right)\right) M_{12}+M_{12}^{\prime} \mathbf{G} \Lambda\left(\dot{\delta}_{k}(\tau)\right) M_{2}\right. \\
& \left.+M_{3}^{\prime}\left(G_{0}+\mathbf{G} \Lambda\left(\sigma_{k}(0)+\delta_{k}(\tau)\right)\right) M_{2}\right\}+M_{12}^{\prime} \mathbf{F} \Lambda\left(\dot{\delta}_{k}(\tau)\right) M_{12}, \\
\Pi_{3}\left(\sigma_{k}(0)\right)= & M_{24}^{\prime} X\left(\sigma_{k}(0)\right) M_{24} .
\end{aligned}
$$

Moreover, from (20) one has

$$
\left(Y_{1} x_{k}(\tau)+Y_{2} \dot{x}_{k}(\tau)\right)^{\prime}\left[A\left(\sigma_{k}(\tau)\right) x_{k}(\tau)+B \psi_{k}-\dot{x}_{k}(\tau)+B K\left(\sigma_{k}(0)\right) x_{k}(0)\right]=0,
$$

for any matrices $Y_{1}$ and $Y_{2}$ of appropriate dimensions. Define now

$$
\Pi_{1}\left(\sigma_{k}(0), \delta_{k}(\tau), \dot{\delta}_{k}(\tau)\right)=\hat{\Pi}_{1}\left(\sigma_{k}(0), \delta_{k}(\tau), \dot{\delta}_{k}(\tau)\right)+H e\left\{\Theta\left(\sigma_{k}(0), \delta_{k}(\tau)\right)\right\},
$$

with

$$
\begin{aligned}
\Theta\left(\sigma_{k}(0), \delta_{k}(\tau)\right)=\left(Y_{1} M_{1}+Y_{2} M_{3}\right)^{\prime}\left(\left(A_{0}+\mathbf{A} \Lambda\left(\sigma_{k}(0)+\delta_{k}(\tau)\right)\right)\right. & M_{1} \\
& \left.+B\left(K_{0}+\mathbf{K} \Lambda\left(\sigma_{k}(0)\right)\right) M_{2}-M_{3}+B M_{4}\right) .
\end{aligned}
$$

In order to eliminate the products between variables (such as the ones between matrices $Y_{1}$ and $Y_{2}$ and $\left.\left(K_{0}+\mathbf{K} \Lambda\left(\sigma_{k}(0)\right)\right)\right)$ and to obtain conditions in LMI form, aiming at guaranteeing that $\dot{\mathscr{W}}<0$, we perform now a congruence transformation. With this aim, consider $Y_{1}=\varepsilon Y_{2}$ and suppose now that $Y_{2}$ is nonsingular, $\bar{Y}=Y_{2}^{-1}$ and $\bar{U}\left(\sigma_{k}(0)\right)=U\left(\sigma_{k}(0)\right)^{-1}=\bar{U}_{0}+\bar{U}\left[\sigma_{k}(0) \otimes I\right]$. Thus, defining $\tilde{\chi}(\tau)=\Xi^{-1} \chi(\tau)$, with $\Xi=\operatorname{diag}\left\{\bar{Y}, \bar{Y}, \bar{Y}, \bar{U}\left(\sigma_{k}(0)\right)\right\}$, and recalling that $Z=\bar{Z}^{\prime} \bar{Z}$, it is possible to rewrite (41) as follows:

$$
\begin{array}{r}
\dot{\mathscr{W}}+\left\|x_{k}(\tau)\right\|_{Z}^{2}+\left\|\operatorname{sat}\left(u_{k}(\tau)\right)\right\|_{H}^{2}-2 \psi_{k}^{\prime} U\left(\sigma_{k}(0)\right)\left(\psi_{k}+S\left(\sigma_{k}(0)\right) x_{k}(0)\right) \leq \\
\tilde{\chi}^{\prime}(\tau)\left[\Xi^{\prime} \Pi_{1}\left(\sigma_{k}(0), \delta_{k}(\tau), \dot{\delta}_{k}(\tau)\right) \Xi+\left(T_{k}-\tau\right) \Xi^{\prime} \Pi_{2}\left(\sigma_{k}(0), \delta_{k}(\tau), \dot{\delta}_{k}(\tau)\right) \Xi\right. \\
+\left(T_{k}-2 \tau\right) \Xi^{\prime} \Pi_{3}\left(\sigma_{k}(0)\right) \Xi+\tau \Xi^{\prime}\left(Q_{0}+\mathbf{Q} \Lambda\left(\sigma_{k}(0)+\delta_{k}(\tau)\right)\right) R^{-1}\left(Q_{0}+\mathbf{Q} \Lambda\left(\sigma_{k}(0)+\delta_{k}(\tau)\right)\right)^{\prime} \Xi \\
\left.+\Xi^{\prime} M_{1}^{\prime} \bar{Z}^{\prime} \bar{Z} M_{1} \Xi+\Xi^{\prime}\left(M_{2}^{\prime}\left(K_{0}+\mathbf{K} \Lambda\left(\sigma_{k}(0)\right)\right)^{\prime}+M_{4}^{\prime}\right) H\left(\left(K_{0}+\mathbf{K} \Lambda\left(\sigma_{k}(0)\right)\right) M_{2}+M_{4}\right) \Xi\right] \tilde{\chi}(\tau) \\
=\tilde{\chi}^{\prime}(\tau) \Psi\left(\sigma_{k}(0), \delta_{k}(\tau), \dot{\delta}_{k}(\tau), \tau, T_{k}\right) \tilde{\chi}(\tau) .
\end{array}
$$

From (44), if the condition

$$
\Psi\left(\sigma_{k}(0), \delta_{k}(\tau), \dot{\delta}_{k}(\tau), \tau, T_{k}\right)<0
$$

holds for all $\sigma_{k}(0) \in \mathscr{B}_{\sigma}, \delta_{k}(\tau) \in \mathscr{B}_{\delta_{k}}, \dot{\delta}_{k}(\tau) \in \mathscr{B}_{\dot{\delta}}, \tau \in\left[0, T_{k}\right)$ and $T_{k} \in\left[\mathscr{T}_{1}, \mathscr{T}_{2}\right]$ and provided that $\Xi$ is nonsingular, it follows that $\left(\Xi^{-1}\right)^{\prime} \Psi\left(\sigma_{k}(0), \delta_{k}(\tau), \dot{\delta}_{k}(\tau), \tau, T_{k}\right) \Xi^{-1}<0$. In this case, let the maximal eigenvalue of $\left(\Xi^{-1}\right)^{\prime} \Psi\left(\sigma_{k}(0), \delta_{k}(\tau), \dot{\delta}_{k}(\tau), \tau, T_{k}\right) \Xi^{-1}$, considering all admissible values for $\sigma_{k}(0), \delta_{k}(\tau)$ and $\dot{\delta}_{k}(\tau)$, be denoted by $\lambda_{\max }$ Thus, there exists a positive scalar $\mu=-\lambda_{\max }$ such that:

$$
\begin{aligned}
\tilde{\chi}^{\prime}(\tau) \Psi\left(\sigma_{k}(0), \delta_{k}(\tau), \dot{\delta}_{k}(\tau), \tau, T_{k}\right) \tilde{\chi}(\tau) & =\chi^{\prime}(\tau)\left(\left(\Xi^{-1}\right)^{\prime} \Psi\left(\sigma_{k}(0), \delta_{k}(\tau), \dot{\delta}_{k}(\tau), \tau, T_{k}\right) \Xi^{-1}\right) \chi(\tau) \\
& \leq-\mu\|\chi(\tau)\|^{2} \leq-\mu\left\|\begin{array}{l}
x_{k}(\tau) \\
x_{k}(0)
\end{array}\right\|^{2},
\end{aligned}
$$


and, consequently, condition (24) of Theorem 1 holds with $p=2$.

We show now that (31) and (32) imply that (45) holds for all $\sigma_{k}(0) \in \mathscr{B}_{\sigma}, \delta_{k}(\tau) \in \mathscr{B}_{\delta_{k}}, \dot{\delta}_{k}(\tau) \in \mathscr{B}_{\dot{\delta}}$, $\tau \in\left[0, T_{k}\right)$ and $T_{k} \in\left[\mathscr{T}_{1}, \mathscr{T}_{2}\right]$. Applying Schur's complement to (45) and multiplying the result by $\gamma_{k}$, it follows that (45) is equivalent to

$$
\begin{gathered}
\tilde{\Psi}\left(\sigma_{k}(0), \delta_{k}(\tau), \dot{\delta}_{k}(\tau), \tau, T_{k}\right)= \\
{\left[\begin{array}{cccc}
\Pi & \tau\left(\tilde{Q}_{0}+\tilde{\mathbf{Q}} \Lambda\left(\sigma_{k}(0)+\delta_{k}(\tau)\right)\right) & \left(\tilde{Y} M_{1}\right)^{\prime} \bar{Z}^{\prime} & \left(\left(\tilde{K}_{0}+\tilde{\mathbf{K}} \Lambda\left(\sigma_{k}(0)\right)\right) M_{2}+\left(\tilde{U}_{0}+\tilde{\mathbf{U}} \Lambda(v)\right) M_{4}\right)^{\prime} \\
* & -\tau \tilde{R} & 0 & 0 \\
* & * & -\gamma_{k} I & 0 \\
* & * & * & -\gamma_{k} H^{-1}
\end{array}\right]<0,}
\end{gathered}
$$

where

$$
\begin{array}{r}
\Pi=\Pi\left(\sigma_{k}(0), \delta_{k}(\tau), \dot{\delta}_{k}(\tau), \tau, T_{k}\right)=\tilde{\Pi}_{1}\left(\sigma_{k}(0), \delta_{k}(\tau), \dot{\delta}_{k}(\tau)\right)+\left(T_{k}-\tau\right) \tilde{\Pi}_{2}\left(\sigma_{k}(0), \delta_{k}(\tau), \dot{\delta}_{k}(\tau)\right) \\
+\left(T_{k}-2 \tau\right) \tilde{\Pi}_{3}\left(\sigma_{k}(0)\right),
\end{array}
$$

with matrices defined in (42) and the following change of variables:

$$
\begin{gathered}
\tilde{Y}=\gamma_{k} \bar{Y}, \tilde{\Xi}=\gamma_{k} \Xi, \tilde{P}_{j}=\gamma_{k}^{-1} \tilde{Y}^{\prime} P_{j} \tilde{Y}, \tilde{F}_{j}=\gamma_{k}^{-1} \tilde{Y}^{\prime} F_{j} \tilde{Y}, \tilde{G}_{j}=\gamma_{k}^{-1} \tilde{Y}^{\prime} G_{j} \tilde{Y}, \tilde{Q}_{j}=\gamma_{k}^{-1} \tilde{\Xi}^{\prime} Q_{j} \tilde{Y} \\
\tilde{X}\left(\sigma_{k}(0)\right)=\tilde{X}_{0}+\Lambda\left(\sigma_{k}(0)\right)=\left[\begin{array}{cc}
\tilde{Y} & 0 \\
0 & \tilde{U}\left(\sigma_{k}(0)\right)
\end{array}\right]^{\prime} \gamma_{k}^{-1} X\left(\sigma_{k}(0)\right)\left[\begin{array}{cc}
\tilde{Y} & 0 \\
0 & \tilde{U}\left(\sigma_{k}(0)\right)
\end{array}\right], \\
\tilde{S}_{j}=S_{j} \tilde{Y}, \tilde{U}\left(\sigma_{k}(0)\right)=\gamma_{k} U\left(\sigma_{k}(0)\right)^{-1}, \quad \tilde{K}_{j}=K_{j} \tilde{Y}, \quad \tilde{R}=\gamma_{k}^{-1} \tilde{Y}^{\prime} R \tilde{Y} .
\end{gathered}
$$

Then, provided that $\Xi$ is invertible, a sufficient condition to verify (24) is given by the matrix inequality (47). Furthermore, as $\tilde{\Psi}\left(\sigma_{k}(0), \delta_{k}(\tau), \dot{\delta}_{k}(\tau), \tau, T_{k}\right)$ depends affinely on $\sigma_{k}(0), \delta_{k}(\tau)$ and $\dot{\delta}_{k}(\tau)$, by convexity, a necessary and sufficient condition to ensure (47) consists in satisfying this inequality at the vertices of polytopes $\mathscr{B}_{\sigma}, \mathscr{B}_{\delta_{k}}$ and $\mathscr{B}_{\dot{\delta}}$. We should therefore verify

$$
\tilde{\Psi}\left(v, \beta, \eta, \tau, T_{k}\right)<0,
$$

i.e.

$$
\left[\begin{array}{cccc}
\Pi\left(v, \beta, \eta, \tau, T_{k}\right) & \tau\left(\tilde{Q}_{0}+\tilde{\mathbf{Q}} \Lambda(v+\beta)\right) & \left(\tilde{Y} M_{1}\right)^{\prime} \bar{Z}^{\prime} & \left(\left(\tilde{K}_{0}+\tilde{\mathbf{K}} \Lambda(v)\right) M_{2}+\left(\tilde{U}_{0}+\tilde{\mathbf{U}} \Lambda(v)\right) M_{4}\right)^{\prime} \\
* & -\tau \tilde{R} & 0 & 0 \\
* & * & -\gamma_{k} I & 0 \\
* & * & * & -\gamma_{k} H^{-1}
\end{array}\right]<0,
$$

for all $(\nu, \beta, \eta) \in \operatorname{Ver}\left(\mathscr{B}_{\sigma}\right) \times \operatorname{Ver}\left(\mathscr{B}_{\delta_{k}}\right) \times \operatorname{Ver}\left(\mathscr{B}_{\dot{\delta}}\right)$. Moreover, as (50) is affine in $\tau$ and $T_{k}$, by applying convexity arguments twice, we conclude that the verification of (31) and (32) for $r=1,2$ is equivalent to satisfy (50), $\forall \tau \in\left[0, T_{k}\right], \forall T_{k} \in\left[\mathscr{T}_{1}, \mathscr{T}_{2}\right]$.

Note now that, if $(32)$ is verified, it follows that $\tilde{\Pi}_{1}(v, \beta, \eta)-\mathscr{T}_{r} \tilde{\Pi}_{3}(v)<0$. Then, from the definition of $\tilde{\Pi}_{1}(v, \beta, \eta)$ and $\tilde{\Pi}_{3}(v)$ in (37), and taking into account that $M_{3} M_{1}^{\prime}=M_{3} M_{2}^{\prime}=M_{3} M_{4}^{\prime}=$ $M_{3} M_{12}^{\prime}=0$ and $M_{3}^{\prime} M_{24}=0$, it follows that:

$$
M_{3}\left(\tilde{\Pi}_{1}(v, \beta, \eta)-\mathscr{T}_{r} \tilde{\Pi}_{3}(v)\right) M_{3}^{\prime}=-\tilde{Y}-\tilde{Y}^{\prime}<0,
$$

which implies that, if (32) is verified, $\tilde{Y}$ is a nonsingular matrix. Moreover, from (34), one has that $\tilde{U}\left(\sigma_{k}(0)\right)$ is nonsingular for all $\sigma_{k}(0) \in \mathscr{B}_{\sigma}$. Hence, we conclude that matrix $\Xi$ is nonsingular. Thus, 
the satisfaction of (31) and (32) for $r=1,2$ indeed ensures that (46) holds for some $\mu>0$ and the condition (24) of Theorem 1 is satisfied.

As (33) is verified $\forall v \in \operatorname{Ver}\left(\mathscr{B}_{\sigma}\right)$, by right and left-multiplying (33) by $\operatorname{diag}\left\{\tilde{Y}^{-1}, I\right\}$ and its transpose, respectively, and then using convexity arguments, it follows that

$$
\left[\begin{array}{cc}
\frac{\left(P_{0}+\mathbf{P} \Lambda\left(\sigma_{k}(0)\right)\right)}{\gamma_{k}} & \left(K_{0}+\mathbf{K} \Lambda\left(\sigma_{k}(0)\right)\right)_{(i)}^{\prime}-\left(S_{0}+\mathbf{S} \Lambda\left(\sigma_{k}(0)\right)\right)_{(i)}^{\prime} \\
* & \bar{u}_{(i)}^{2}
\end{array}\right]>0,
$$

which, from Schur's complement, implies that condition (23) of Theorem 1 is satisfied (see for instance [26] for details).

Finally, (35) ensures that $P\left(\sigma_{k}(\tau)\right)>0$ for all $\sigma_{k}(\tau) \in \mathscr{B}_{\sigma}$. Hence, we conclude that if (31)-(35) are satisfied, conditions (21)-(24) of Theorem 1 are verified for all $\tau \in\left[0, T_{k}\right), \forall T_{k} \in\left[\mathscr{T}_{1}, \mathscr{T}_{2}\right]$ and $\forall \sigma_{k}(\tau) \in \mathscr{B}_{\sigma}, \forall \dot{\sigma}_{k}(\tau) \in \mathscr{B}_{\dot{\delta}}$, and thus $(i)$ and (ii) hold, i.e. $\lim _{t \rightarrow \infty} x(t)=0$ and $J\left(t_{k}, \infty\right) \leq \gamma_{k}$, provided $x\left(t_{k}\right) \in \mathscr{L}_{V}\left(\gamma_{k}\right)$.

Remark 2. For simplicity, we have considered matrix B parameter-independent in the developments. The parameter-dependent case, i.e. when $B(\sigma(t))=B_{0}+\sum_{j=1}^{N} \sigma_{(j)}(t) B_{j}=B_{0}+B \Lambda(\sigma(t))$, with $\boldsymbol{B}=\left[\begin{array}{lll}B_{1} & \ldots & B_{N}\end{array}\right]$, can also be addressed, but with more involved conditions. Note that in this case, terms $B\left(\sigma_{k}(0)+\delta_{k}(\tau)\right) \tilde{K}\left(\sigma_{k}(0)\right)$ and $B\left(\sigma_{k}(0)+\delta_{k}(\tau)\right) \tilde{U}\left(\sigma_{k}(0)\right)$ will appear in matrix $\Pi$ in (47), leading to products $B\left(\sigma_{k}(0)\right) \tilde{K}\left(\sigma_{k}(0)\right)$ and $B\left(\sigma_{k}(0)\right) \tilde{U}\left(\sigma_{k}(0)\right)$. Due to these products, convexity arguments cannot be applied directly. To overcome this issue, the relaxation technique considered in [31] can be applied, leading to two additional sets of matrix inequalities to be verified. Roughly speaking, we should replace conditions (31) and (32) by the following ones:

$$
\begin{aligned}
& \Psi_{1}\left(v_{f}, v_{f}, \beta_{g}, \eta_{h}, \mathscr{T}_{r}\right)<0, \\
& \frac{2}{N-1} \Psi_{1}\left(v_{f}, v_{f}, \beta_{g}, \eta_{h}, \mathscr{T}_{r}\right)+\Psi_{1}\left(v_{f}, v_{p}, \beta_{g}, \eta_{h}, \mathscr{T}_{r}\right)+\Psi_{1}\left(v_{p}, v_{f}, \beta_{g}, \eta_{h}, \mathscr{T}_{r}\right)<0, \\
& \Psi_{2}\left(v_{f}, v_{f}, \beta_{g}, \eta_{h}, \mathscr{T}_{r}\right)<0, \\
& \frac{2}{N-1} \Psi_{2}\left(v_{f}, v_{f}, \beta_{g}, \eta_{h}, \mathscr{T}_{r}\right)+\Psi_{2}\left(v_{f}, v_{p}, \beta_{g}, \eta_{h}, \mathscr{T}_{r}\right)+\Psi_{2}\left(v_{p}, v_{f}, \beta_{g}, \eta_{h}, \mathscr{T}_{r}\right)<0,
\end{aligned}
$$

for $f=1, \ldots, 2^{N}, p=1, \ldots, 2^{N}, p \neq f, g=1, \ldots, 2^{N}, h=1, \ldots, 2^{N}, r=1,2$, where $\Psi_{1}\left(v, \phi, \beta, \eta, \mathscr{T}_{r}\right)$ and $\Psi_{2}\left(v, \phi, \beta, \eta, \mathscr{T}_{r}\right)$ corresponds, respectively, to $\Psi_{1}$ in (31) and $\Psi_{2}$ in (32), with $B$ replaced by $B_{0}+B \Lambda(\phi+\beta)$.

\section{LPV-MPC Control Law}

In this section, we detail Step 3 of Algorithm 1 by using the results provided in Section 3 to solve Problem 1. Then, we show that the LPV-MPC law implemented as described in Algorithm 1 leads to the asymptotic stability of the origin of the closed-loop system. Moreover, if at instant $k=0$ there exists a feasible solution to the optimization problem to be solved in Step 3, then the feasibility is guaranteed for all $k \in \mathbb{N}$.

Let us recall that the basic idea of Algorithm 1 is to compute a new feedback matrix $K_{k}\left(\sigma\left(t_{k}\right)\right)$ at each sampling time $t_{k}$ in order to minimize an upper bound $\gamma_{k}$ on $J\left(t_{k}, \infty\right)$, based on the measurements 
of $x\left(t_{k}\right)$ and $\sigma\left(t_{k}\right)$. Hence, from the results stated in Theorem 2, in Step 3 of Algorithm 1 the following optimization problem is considered to compute $K_{k}\left(\sigma\left(t_{k}\right)\right)$ :

$$
\begin{aligned}
& \min \gamma_{k} \\
& \text { subject to } \\
& {\left[\begin{array}{cc}
1 & x_{k}(0)^{\prime} \\
x_{k}(0) & \tilde{Y}_{k}+\tilde{Y}_{k}^{\prime}-\tilde{P}_{k, 0}-\tilde{\mathbf{P}}_{k} \Lambda(v)
\end{array}\right] \geq 0,} \\
& \Psi_{k, 1}\left(v, \beta, \eta, \mathscr{T}_{r}\right)<0 \text {, } \\
& \Psi_{k, 2}\left(v, \beta, \eta, \mathscr{T}_{r}\right)<0 \text {, } \\
& {\left[\begin{array}{cc}
\tilde{P}_{k, 0}+\tilde{\mathbf{P}}_{k} \Lambda(v) & \left(\tilde{K}_{k, 0}+\tilde{\mathbf{K}}_{k} \Lambda(v)\right)_{(i)}^{\prime}-\left(\left(\tilde{S}_{k, 0}+\tilde{\mathbf{S}}_{k} \Lambda(v)\right)\right)_{(i)}^{\prime} \\
* & \bar{u}_{(i)}^{2}
\end{array}\right] \geq 0,} \\
& \text { for all }(v, \beta, \eta) \in \operatorname{Ver}\left(\mathscr{B}_{\sigma}\right) \times \operatorname{Ver}\left(\mathscr{B}_{\delta_{k}}\right) \times \operatorname{Ver}\left(\mathscr{B}_{\dot{\delta}}\right), \forall i=1, \ldots, m \text { and } \forall r=1,2 \text {, }
\end{aligned}
$$

with $\tilde{P}_{k, j}, \tilde{F}_{k, j}, \tilde{G}_{k, j}, \tilde{K}_{k, j}, \tilde{S}_{k, j}, \tilde{Q}_{k, j}, \tilde{U}_{k, j}, \tilde{X}_{k, j}, \tilde{Y}_{k}, \tilde{R}_{k}$ and $\varepsilon$ as decision variables. The subscript " $k$ " in the variable matrices stands for the corresponding matrix computed at sampling instant $t_{k}$. From Lemma 1 in [27] and considering the change of variables defined in (48), the first matrix inequality in (56) ensures that

$$
x_{k}(0)^{\prime}\left(P_{k, 0}+\mathbf{P}_{k} \Lambda(v)\right) x_{k}(0)=x_{k}(0)^{\prime} P_{k}(v) x_{k}(0) \leq \gamma_{k},
$$

for all $v \in \operatorname{Ver}\left(\mathscr{B}_{\sigma}\right)$ and, by convexity, we conclude that $x_{k}(0)=x\left(t_{k}\right) \in \mathscr{L}_{V}\left(\gamma_{k}\right)$. Hence, from Theorem 2, it follows that $J\left(t_{k}, \infty\right)<V\left(x_{k}(0), \sigma_{k}(0)\right)=x_{k}(0)^{\prime} P_{k}\left(\sigma_{k}(0)\right) x_{k}(0)=x\left(t_{k}\right) P_{k}\left(\sigma\left(t_{k}\right)\right) x\left(t_{k}\right) \leq \gamma_{k}$.

Thus, at each sampling instant $t=t_{k}$, state feedback gains $K_{k, j}=\tilde{K}_{k, j} \tilde{Y}_{k}^{-1}$ for $j=0, \ldots, N$ are computed to minimize $\gamma_{k}$ and to ensure that the closed-loop system trajectories converge to the origin. At this point, two important issues arise from the application of Algorithm 1: the optimization problem (56) must be feasible at each instant and it should be ensured that $\lim _{t \rightarrow \infty} x(t)=0$. Theorems 3 and 4 in the sequel address these issues.

Theorem 3. If the optimization problem (56) is feasible for $k=0$, then it is feasible for all $k>0$.

Proof. The first matrix inequality in (56) ensures that $x_{k}(0)=x\left(t_{k}\right) \in \mathscr{L}_{V}\left(\gamma_{k}\right)$. Hence from Theorems 1 and 2, it follows that

$$
\Delta V(k)=x^{\prime}\left(t_{k+1}\right) P_{k}\left(\sigma\left(t_{k+1}\right)\right) x\left(t_{k+1}\right)-x^{\prime}\left(t_{k}\right) P_{k}\left(\sigma\left(t_{k}\right)\right) x\left(t_{k}\right) \leq-\mathscr{T}_{1} \mu\left\|x\left(t_{k}\right)\right\|^{2},
$$

with $P_{k}(\sigma)=P_{k, 0}+\mathbf{P}_{k} \Lambda(\sigma), P_{k, j}=\gamma_{k}\left(\tilde{Y}_{k}^{\prime}\right)^{-1} \tilde{P}_{k, j} \tilde{Y}_{k}^{-1}, j=0, \ldots, N$. Then we can conclude that

$$
x^{\prime}\left(t_{k+1} \mid t_{k}\right) P_{k}\left(\sigma\left(t_{k+1}\right)\right) x\left(t_{k+1} \mid t_{k}\right)<x^{\prime}\left(t_{k} \mid t_{k}\right) P_{k}\left(\sigma\left(t_{k}\right)\right) x\left(t_{k} \mid t_{k}\right) \leq \gamma_{k}
$$

which ensures that the optimal solution of (56) at $t=t_{k}$ is a feasible solution for $t=t_{k+1}$. Repeating this reasoning, we conclude that this solution at $t=t_{k}$ is a feasible one at $t_{k+s+1}, \forall s \geq 0$.

It is worth noticing that the value of $\gamma_{k}$ depends on the measured value of the state at the instant $t_{k}$, i.e. $x\left(t_{k} \mid t_{k}\right)=x\left(t_{k}\right)$. Furthermore, from (59), we can conclude that $\gamma_{k}$ always decreases as $k$ increases.

Theorem 4. The sampled-data LPV-MPC law computed by Algorithm 1, with Step 3 given by the solution to optimization problem (56), ensures that the trajectories of the closed-loop system converge to the origin as $t \rightarrow \infty$, provided that (56) is feasible for $k=0$. 
Proof. Let $P_{k, j}=\gamma_{k}\left(\tilde{Y}_{k}^{\prime}\right)^{-1} \tilde{P}_{k, j} \tilde{Y}_{k}^{-1}, j=0, \ldots, N$ and $\gamma_{k}$ obtained from the optimal solution to (56) at instant $t=t_{k}$ and $P_{k}(\sigma)=P_{k, 0}+\mathbf{P}_{k} \Lambda(\sigma)$. From Theorem 3, as the solution to (56) at instant $t=t_{k}$ is feasible for $t=t_{k+1}$, from (59) it follows that:

$$
x^{\prime}\left(t_{k+1} \mid t_{k}\right) P_{k}\left(\sigma\left(t_{k+1}\right)\right) x\left(t_{k+1} \mid t_{k}\right)=\tilde{\gamma}_{k+1}<\gamma_{k},
$$

where $\tilde{\gamma}_{k+1}$ is probably a non-optimal solution to (56). Consider now $P_{k+1, j}=\gamma_{k+1}\left(\tilde{Y}_{k+1}^{\prime}\right)^{-1} \tilde{P}_{k+1, j} \tilde{Y}_{k+1}^{-1}$, $j=0, \ldots, N$ and $\gamma_{k+1}$ obtained from the optimal solution to (56) at instant $t=t_{k+1}$ and $x\left(t_{k+1} \mid t_{k}\right)=$ $x\left(t_{k+1} \mid t_{k+1}\right)$. Thus, by the optimality principle, from (60) one has that

$$
x^{\prime}\left(t_{k+1} \mid t_{k+1}\right) P_{k+1}\left(\sigma\left(t_{k+1}\right)\right) x\left(t_{k+1} \mid t_{k+1}\right)=\gamma_{k+1} \leq \tilde{\gamma}_{k+1}=x^{\prime}\left(t_{k+1} \mid t_{k+1}\right) P_{k}\left(\sigma\left(t_{k+1}\right)\right) x\left(t_{k+1} \mid t_{k+1}\right)<\gamma_{k} .
$$

Furthermore, since from (58) one has

$$
x^{\prime}\left(t_{k+1} \mid t_{k+1}\right) P_{k}\left(\sigma\left(t_{k+1}\right)\right) x\left(t_{k+1} \mid t_{k+1}\right)-x^{\prime}\left(t_{k} \mid t_{k}\right) P_{k}\left(\sigma\left(t_{k}\right)\right) x\left(t_{k} \mid t_{k}\right)<-\mathscr{T}_{1} \mu\left\|x\left(t_{k} \mid t_{k}\right)\right\|^{2},
$$

it follows from (61) that

$$
x^{\prime}\left(t_{k+1} \mid t_{k+1}\right) P_{k+1}\left(\sigma\left(t_{k+1}\right)\right) x\left(t_{k+1} \mid t_{k+1}\right)-x^{\prime}\left(t_{k} \mid t_{k}\right) P_{k}\left(\sigma\left(t_{k}\right)\right) x\left(t_{k} \mid t_{k}\right)<-\mathscr{T}_{1} \mu\left\|x\left(t_{k} \mid t_{k}\right)\right\|^{2}, \forall k \geq 0 .
$$

Then, we can conclude that $x^{\prime}\left(t_{k} \mid t_{k}\right) P_{k}\left(\sigma\left(t_{k}\right)\right) x\left(t_{k} \mid t_{k}\right) \rightarrow 0$ as $k \rightarrow \infty$. Hence, since $J\left(t_{k}, \infty\right) \leq$ $x^{\prime}\left(t_{k} \mid t_{k}\right) P_{k}\left(\sigma\left(t_{k}\right)\right) x\left(t_{k} \mid t_{k}\right)$, it follows that $\lim _{k \rightarrow \infty} J\left(t_{k}, \infty\right)=0$, which implies that $x(t) \rightarrow 0$ as $t \rightarrow \infty$.

Remark 3. The numerical complexity of Algorithm 1 is related to the optimization problem (56), which has to be solved at each sampling time. It should be noticed that for a fixed $\varepsilon$ this problem is convex, since all the constraints are LMIs. The numerical complexity that LMI solvers are able to handle is an open topic, which is discussed in some references only when the authors find convergence problems. It usually depends on the considered solver (e.g., LMILAB, SeDuMi or MOSEK) and some optimization problem parameters such as number of LMI conditions $\left(n_{l}\right)$, maximum LMI order $\left(n_{o}\right)$ and number of decision variables $\left(n_{v}\right)$. Based on the LMI conditions in (56), these parameters can be computed as function of the plant dimensions ( $n, m$ and $N)$. On the other hand, the complexity of the conditions in the present problem can be reduced by considering parameterindependent versions of function $V$ and/or looped-functional $\mathscr{V}_{0}$, at the expense of more conservative solutions. In this case, one should consider $\tilde{P}_{k}(\sigma)=\tilde{P}_{k, 0}, \tilde{F}_{k}(\sigma)=\tilde{F}_{k, 0}, \tilde{G}_{k}(\sigma)=\tilde{G}_{k, 0}, \tilde{S}_{k}(\sigma)=\tilde{S}_{k, 0}$, $\tilde{Q}_{k}(\sigma)=\tilde{Q}_{k, 0}, \tilde{U}_{k}(\sigma)=\tilde{U}_{k, 0}$ and $\tilde{X}_{k}(\sigma)=\tilde{X}_{k, 0}$, i.e. assume $\tilde{\mathbf{P}}=0, \tilde{\mathbf{F}}=0, \tilde{\mathbf{G}}=0, \tilde{\mathbf{S}}=0, \tilde{\mathbf{Q}}=0, \tilde{\mathbf{U}}=0$ and $\tilde{\mathbf{X}}=0$. For comparison purposes, the expressions of $n_{l}, n_{o}$ and $n_{v}$ are shown in Table 1 for both cases.

Table 1: Numerical complexity associated to optimization problem (56).

\begin{tabular}{ccc}
\hline & Parameter-dependent conditions & Parameter-independent conditions \\
\hline$n_{v}$ & $\begin{array}{c}(5.5 N+7) n^{2}+(1.5 N+2+(4 N+4) m) n \\
+(0.5 N+0.5) m^{2}+(1.5 N+1.5) m+1\end{array}$ & $7 n^{2}+(2+(4+N) m) n+0.5 m^{2}$ \\
& $2^{3 N+2}+2^{2 N}+(m+2) 2^{N}$ & $+1.5 m+1$ \\
\hline$n_{l}$ & $5 n+2 m$ & $2^{2 N+2}+2^{N} m+1$ \\
\hline$n_{o}$ & & $5 n+2 m$ \\
\hline
\end{tabular}

It should be noticed that the computation time can also be significantly reduced if we consider the solution obtained in the previous sampling instant as initial guess to problem (56). Note that for two consecutive sampling instants the optimal solution to (56) is not expected to change much. 
Finally, we should point out that, if the solver is not able to find the optimal solution in the available computation time, one can always use the control input computed from the gains obtained in the previous sampling instant, i.e. $u(t)=K_{k-1}\left(\sigma\left(t_{k}\right)\right) x\left(t_{k}\right), \forall t \in\left[t_{k}, t_{k+1}\right)$. From the same arguments employed in the proof of Theorem 4, the convergence of the trajectory to the origin is also guaranteed in this case.

\subsection{Estimation of the Region of Attraction}

A pertinent problem is the determination of an estimate of the region of attraction of the origin of the closed-loop system under the LPV-MPC strategy. This region corresponds, in fact, to all initial states $x(0)=x_{0}(0)$ for which problem (56) is feasible at the instant $k=0$.

On the other hand, a feasible solution of the matrix inequalities in Theorem 2 leads to an LPV control law (5) that ensures the convergence to the origin of all trajectories starting in the set $\mathscr{L}_{V}\left(\gamma_{0}\right)$, for all $\sigma(t)$ such that $\sigma(t) \in \mathscr{B}_{\sigma}, \dot{\sigma}(t) \in \mathscr{B}_{\dot{\delta}}$, considering any sampling sequence satisfying $0<\mathscr{T}_{1} \leq$ $T_{k} \leq \mathscr{T}_{2}$. Thus, a suitable estimate of the region of attraction of the LPV-MPC control law can be done by searching a solution that leads to a maximization of $\mathscr{L}_{V}\left(\gamma_{0}\right)$ in terms of some size criterion. For instance, considering a scalar $\alpha>0$, we can maximize a set $\mathscr{E}(\alpha I, 1)=\left\{x \in \mathbb{R}^{n} \mid x^{\prime} \alpha I x \leq 1\right\}$ such that $\mathscr{E}(\alpha I, 1) \subseteq \mathscr{L}_{V}\left(\gamma_{0}\right)$. This can be accomplished by the solution of the following optimization problem:

$$
\begin{aligned}
& \min \alpha \\
& \text { subject to } \\
& (31)-(35), \\
& {\left[\begin{array}{cc}
\alpha I & \tilde{Y}^{\prime}+\tilde{Y}-\left(\tilde{P}_{0}+\tilde{\mathbf{P}} \Lambda(v)\right)
\end{array}\right]>0,} \\
& \text { for all }(v, \beta, \eta) \in \operatorname{Ver}\left(\mathscr{B}_{\sigma}\right) \times \operatorname{Ver}\left(\mathscr{B}_{\delta_{k}}\right) \times \operatorname{Ver}\left(\mathscr{B}_{\dot{\delta}}\right), \forall i=1, \ldots, m \text { and } \forall r=1,2 .
\end{aligned}
$$

Note that the last matrix inequality in (63) implies that $\mathscr{E}(\alpha I, 1) \subseteq \mathscr{E}\left(P_{0}+\mathbf{P} \Lambda(v), \gamma_{0}\right)$ for all $v \in \operatorname{Ver}\left(\mathscr{B}_{\sigma}\right)$ (see $\left.[27]\right)$, which ensures that $\mathscr{E}(\alpha I, 1) \subseteq \mathscr{L}_{V}\left(\gamma_{0}\right)$.

In problem (63), we can consider $\gamma_{0}$ as a free variable. In this case, the larger admissible set $\mathscr{L}_{V}\left(\gamma_{0}\right)$ will be obtained disregarding performance guarantees. This indeed leads to an estimate of the feasibility region of the LPV-MPC strategy.

\subsection{Estimation of the Maximum Inter-sampling Time}

Another problem of interest regards the determination of an estimate of the maximum bound on the intersampling time $T_{k}$, for which there exists a feasible solution considering that the initial state belongs to a given set $\mathscr{X}_{0} \subset \mathbb{R}^{n}$.

For instance, considering that $\mathscr{X}_{0}$ is a ball with radius $\frac{1}{\sqrt{\alpha}}$, i.e. $\mathscr{X}_{0}=\mathscr{E}(\alpha I, 1)=\left\{x \in \mathbb{R}^{n} \mid x^{\prime} \alpha I x \leq\right.$ $1\}$, the following optimization problem can be considered:

$$
\begin{aligned}
& \max \mathscr{T}_{2} \\
& \text { subject to } \\
& (31)-(35), \\
& {\left[\begin{array}{cc}
\alpha I & \tilde{Y}^{\prime}+\tilde{Y}-\left(\tilde{P}_{0}+\tilde{\mathbf{P}} \Lambda(v)\right)
\end{array}\right]>0,} \\
& \text { for all }(v, \beta, \eta) \in \operatorname{Ver}\left(\mathscr{B}_{\sigma}\right) \times \operatorname{Ver}\left(\mathscr{B}_{\delta_{k}}\right) \times \operatorname{Ver}\left(\mathscr{B}_{\dot{\delta}}\right), \forall i=1, \ldots, m \text { and } \forall r=1,2 .
\end{aligned}
$$

Note that a solution for (64) can be indirectly obtained by iteratively increasing $\mathscr{T}_{2}$ and testing the feasibility of the constraints, with $\mathscr{T}_{2}$ fixed in (31) and (32). 


\section{Numerical Examples}

\subsection{Example 1}

Consider the numerical example adapted from [15], where the LPV system is described by ${ }^{3}$ :

$$
\dot{x}(t)=\left[\begin{array}{cc}
0 & 1 \\
0.1 & 1-1.2 \sigma(t)
\end{array}\right] x(t)+\left[\begin{array}{l}
0 \\
1
\end{array}\right] \operatorname{sat}(u(t)),
$$

with $\bar{u}=1$ and $\sigma(t)=a \sin (b \pi t)$, which leads to $|\sigma| \leq a$ and $|\dot{\sigma}| \leq a b \pi$. We assume that $\mathscr{T}_{1}=10 \mathrm{~ms}$, $\varepsilon=1.115$ and a cost function described by $J\left(t_{k}, \infty\right)$ as in $(4)$, with the matrices $H=0.01$ and $Z=I$.

For $T_{k} \in[10,200] \mathrm{ms}, a=1, b=0.1$, we consider the problem of maximizing of $\mathscr{L}_{V}\left(\gamma_{0}\right)$ given in (63). Figure 1 shows sets $\mathscr{L}_{V}\left(\gamma_{0}\right)$ obtained from the proposed approach and the one from [15] in solid and dash-dotted lines, respectively. It can be seen that the proposed results allow to ensure the MPC algorithm feasibility and asymptotic stability for a larger set of admissible initial conditions. Indeed, consider for instance the initial condition $x(0)=[-0.12-0.43]^{\prime}$, which belongs to the set of admissible initial conditions obtained by our method, but not with the one in [15]. In this case, it follows that our method leads to feasibility at $k=0$, with $\gamma_{0}=38.82$ and gain matrices

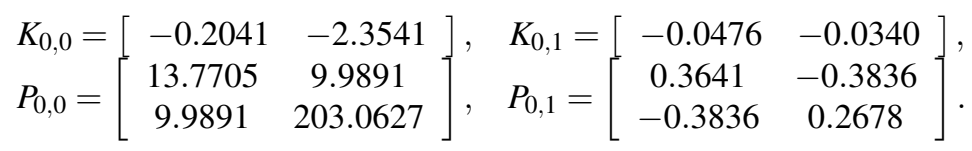

The simulation results are shown in Figure 2. A random sequence of $T_{k}$ given in Figure 5 , where the size of the vertical bars denote the values of $T_{k}$ for the corresponding sampling instants, was considered. On the other hand, conditions of [15] are infeasible in this case.

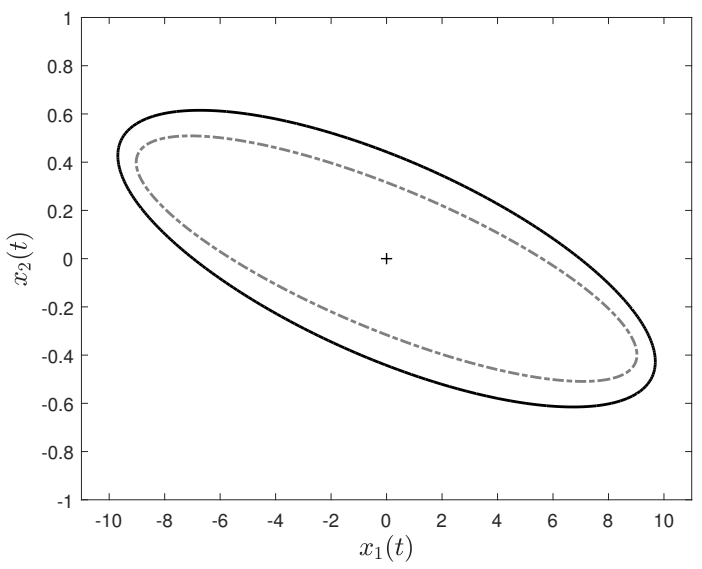

Figure 1: Example $1-\mathscr{L}_{V}\left(\gamma_{0}\right)$ obtained with the proposed approach (solid line) and with the one from in [15] (dash-dotted line).

\footnotetext{
${ }^{3} \operatorname{In}[15]$, the parameter is described by $|\sin (0.01 \pi t)|$, while in this example we consider $\sigma(t)=a \sin (b \pi t)$.
} 


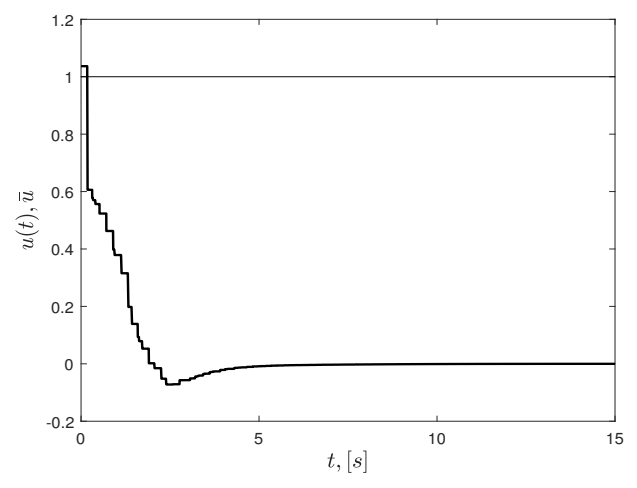

(a) Control input $u(t)$ and bound $\bar{u}$.

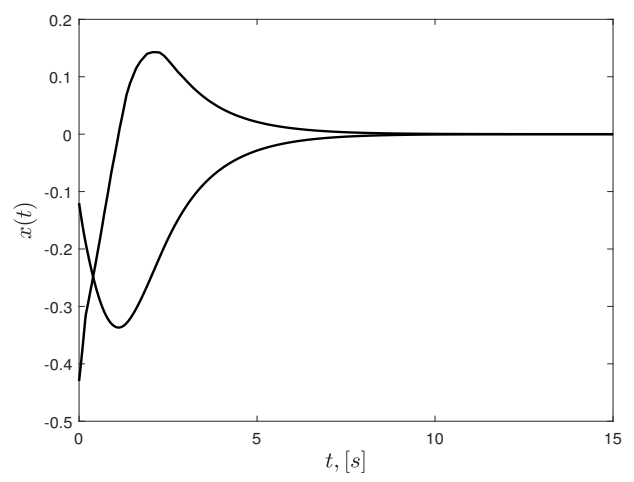

(b) Trajectories of the state vector $x(t)$.

Figure 2: Example 1 - Simulation results for $x(0)=[-0.12-0.43]^{\prime}$ and $T_{k} \in[10,200] \mathrm{ms}$.

Now, the values of $\gamma_{0}$ obtained with the proposed approach and the one from [15], considering $x(0)=\left[\begin{array}{ll}2 & 0\end{array}\right]^{\prime}$, with different values for $a, b$ and two different admissible intervals for $T_{k}$, are compared in Table 2. We can notice that the values of $\gamma_{0}$ obtained from Theorem 2 are smaller than the ones obtained from the conditions proposed in [15]. Moreover, conditions from [15] are not feasible for $|\sigma(t)| \geq 1.5$ and for $T_{k} \in[10,400] \mathrm{ms}$. Note also that the results obtained from [15] do not depend on the bounds of $\dot{\sigma}(t)$ (given indirectly by parameter $b$ ). Matrices $P_{k}$ and $K_{k}$ obtained for $k=0$, considering $T_{k} \in[10,200] \mathrm{ms}, a=1$ and $b=0.1$, are shown in Table 3 for both approaches. It should be pointed out that considering the results in [16] for the same cases shown in Table 2, the conditions are feasible only for $a=0.5$, independently of the time-derivative bound value. In this case, the minimum $\gamma_{0}$ obtained is 50.0094, which is greater than the ones obtained from our method $\left(\gamma_{0}=20.4123\right)$ and from the one in $[15]\left(\gamma_{0}=24.1676\right)$. Note that in [16], the considered control law is a robust one (see Remark 1), i.e. it does not depend on the parameters.

The time responses of $u(t)$ and $x(t)$ obtained with the application of the proposed Algorithm and the one in [15], considering $a=1, b=0.1, T_{k} \in[10,200] \mathrm{ms}$ and the random sequence given in Figure 5, are compared in Figure 3. Note that the convergence to the origin with the proposed approach is faster, which indicates a performance improvement with respect to the approach in [15]. Indeed, it can be observed in Figure 4 that the values of $\gamma_{k}$ obtained with the proposed method are always smaller than the ones from [15].

\subsection{Example 2}

Consider the following LPV model of a single, non-isothermal continuous stirred-tank reactor (CSTR) borrowed from [32] and also treated in [16]:

$$
\begin{aligned}
& \dot{x}_{1}(t)=\left(-1-0.6576 \sigma_{1}(t)\right) x_{1}(t)-0.0094 \sigma_{1}(t) x_{2}(t)+\operatorname{sat}\left(u_{1}(t)\right), \\
& \dot{x}_{2}(t)=0.6576 \times 10^{-8} \sigma_{2}(t) x_{1}(t)+\left(-6.34+0.0094 \times 10^{-8} \sigma_{2}(t)\right) x_{2}(t)-6.0842 \operatorname{sat}\left(u_{2}(t)\right),
\end{aligned}
$$

where the states $x_{1}$ and $x_{2}$ are the product concentration and the temperature inside the tank, respectively, and the control inputs $u_{1}$ and $u_{2}$ correspond to the product feed concentration and the coolant flow, respectively. We consider that the saturation limits are given by $\bar{u}=\left[\begin{array}{ll}1 & 1\end{array}\right]^{\prime}$. The varying 
Table 2: Example 1 - Obtained values of $\gamma_{0}$, for $x(0)=\left[\begin{array}{ll}2 & 0\end{array}\right]^{\prime}, T_{k} \in[10,200] \mathrm{ms}$ and $T_{k} \in[10,400] \mathrm{ms}$.

\begin{tabular}{cc|cc|c}
\hline \multirow{2}{*}{$a$} & \multirow{2}{*}{$b$} & \multicolumn{2}{|c|}{$T_{k} \in[10,200] \mathrm{ms}$} & $T_{k} \in[10,400] \mathrm{ms}^{*}$ \\
\cline { 3 - 5 } & & {$[15]$} & Proposed approach & Proposed approach \\
\hline \hline 0.5 & 0.1 & 24.1676 & 20.4123 & 21.6262 \\
\hline 0.5 & 0.5 & 24.1676 & 21.5500 & 24.6194 \\
\hline 0.5 & 0.9 & 24.1676 & 22.8302 & 28.4927 \\
\hline \hline 1 & 0.1 & 36.9144 & 27.2617 & 30.0190 \\
\hline 1 & 0.5 & 36.9144 & 31.4116 & 45.1375 \\
\hline 1 & 0.9 & 36.9144 & 36.6124 & 73.6624 \\
\hline \hline 1.5 & 0.1 & - & 35.1003 & 40.7236 \\
\hline 1.5 & 0.5 & - & 44.8433 & 109.4602 \\
\hline 1.5 & 0.9 & - & 61.5668 & 237.5040 \\
\hline \hline 2 & 0.1 & - & 43.3198 & 60.8997 \\
\hline 2 & 0.5 & - & 65.6807 & 366.1544 \\
\hline 2 & 0.9 & - & 109.7062 & - \\
\hline$*$
\end{tabular}

${ }^{*}$ For $T_{k} \in[10,400] \mathrm{ms}$ conditions from [15] are not feasible.

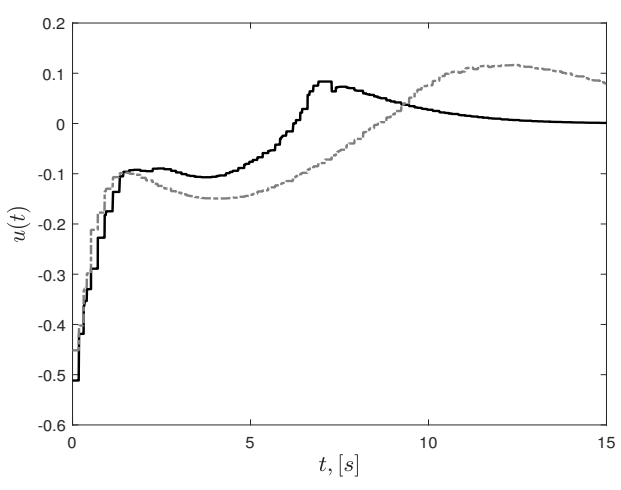

(a) Sampled-data control input $u(t)$.

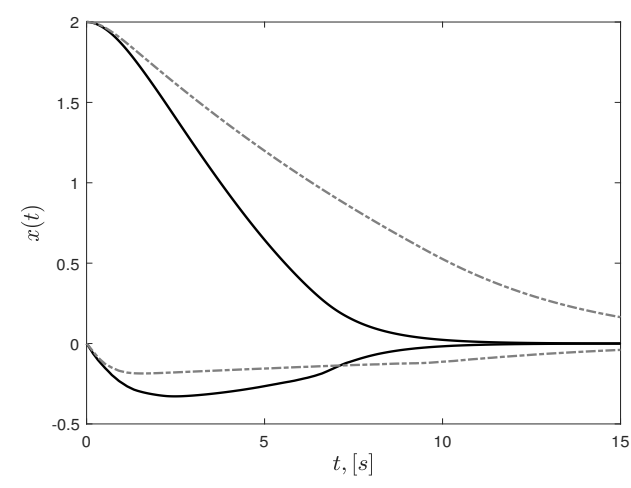

(b) Trajectories of the state vector $x(t)$ starting from $x(0)$.

Figure 3: Example 1 - Simulation results for $x(0)=\left[\begin{array}{ll}2 & 0\end{array}\right]^{\prime}$ and $T_{k} \in\left[\begin{array}{ll}10,200\end{array}\right] \mathrm{ms}$, from Algorithm 1 (solid line) and from Algorithm in [15] (dash-dotted line).

parameters $\sigma_{1}(t)$ and $\sigma_{2}(t)$ are assumed to be bounded in amplitude as follows: $\sigma_{1}(t) \in[1,10]$ and $\sigma_{2}(t) \in[1,100]$. Moreover we assume that $\dot{\sigma}_{1} \in[-1,1]$ and $\dot{\sigma}_{2} \in[-20,20]$.

For comparison purposes, we consider our algorithm and the ones in [15] and [16]. Considering $Z=I, H=0.01 I, T_{k} \in[10,200] \mathrm{ms}, \sigma_{1}(t)=5.5+4.5 \sin ((1 / 4.5) t-\pi), \sigma_{2}(t)=(5.5+4.5 \sin ((1 / 4.5) t-$ $\pi)) \times(5.5+4.5 \sin ((1 / 4.5) t))$ and the initial condition $x(0)=\left[\begin{array}{ll}0.1 & 2\end{array}\right]^{\prime}$, the closed-loop simulation results corresponding to our approach (solid line) and the ones from [15] (dashed line) and [16] (dotted line), are shown in Figure 6 . Table 4 shows the values of $\gamma_{0}$ with each one of the approaches. As it can be observed in Figure 6(c), the convergence of the state to the origin obtained with our approach is faster. Moreover, the guaranteed cost values $\gamma_{k}$ computed at each sampling instant are 


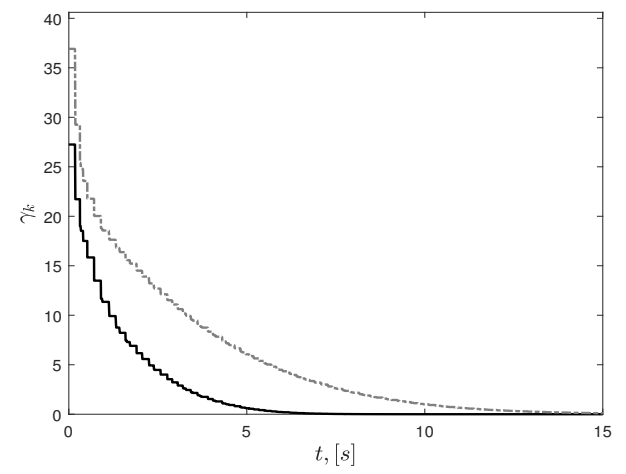

Figure 4: Example 1 - Evolution of $\gamma_{k}$, from Algorithm 1 (solid line) and from Algorithm in [15] (dash-dotted line).

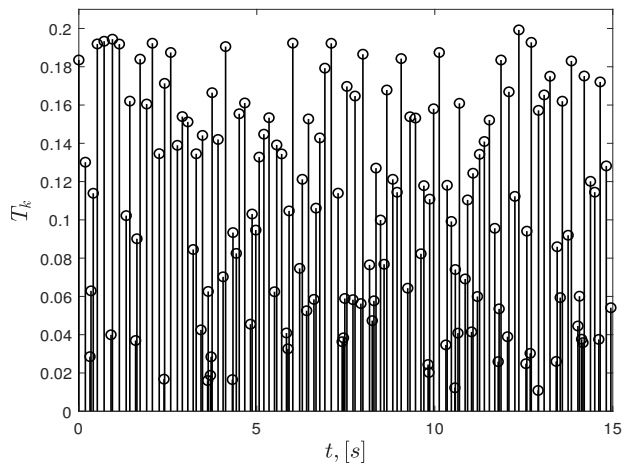

Figure 5: Example 1 - Values of $T_{k}$ for $t=t_{k}$.

Table 3: Example 1 - Matrices $K_{k}$ and $P_{k}$ computed at sampling instant $t_{k}=0$, for $T_{k} \in[10,200] \operatorname{ms}$ and $x(0)=\left[\begin{array}{ll}2 & 0\end{array}\right]^{\prime}$.

\begin{tabular}{cc}
\hline \multicolumn{15}{c}{ Problem $(56)$} \\
\hline$K_{0,0}=\left[\begin{array}{cc}-0.2350 & -2.6965\end{array}\right]$ & $K_{0,0}=\left[\begin{array}{cc}-0.2570 & -2.4033\end{array}\right]$ \\
\hline$K_{0,1}=\left[\begin{array}{ll}-0.0399 & 0.6587\end{array}\right]$ & $K_{0,1}=\left[\begin{array}{cc}-0.1202 & -0.0211\end{array}\right]$ \\
\hline$P_{0}=\left[\begin{array}{cc}9.2286 & 32.6053 \\
32.6053 & 423.5449\end{array}\right]$ & $P_{0,0}=\left[\begin{array}{cc}6.8109 & 18.1534 \\
18.1534 & 171.9655\end{array}\right]$ \\
& $P_{0,1}=\left[\begin{array}{cc}0.0017 & 0.2966 \\
0.2966 & 5.5224\end{array}\right]$ \\
\hline$\gamma_{0}=36.9144$ & $\gamma_{0}=27.2617$
\end{tabular}

depicted in Figure 6(d) for the three cases. Our approach leads to smaller values, which confirms the performance improvement.

It should be pointed out that in [15] and [16], basically the same functional is used to derive the stabilizing conditions. This functional can be seen as the combination of a particular version 
Table 4: Example 2 - value of $\gamma_{0}$

\begin{tabular}{c|c|c}
\hline Problem (56) & {$[15]$} & {$[16]$} \\
\hline 0.3561 & 0.807 & 0.831 \\
\hline
\end{tabular}

of our function (16) and looped-functional (30), where $P\left(\sigma_{k}(\tau)\right)=P_{0}, R=P_{0}, F\left(\sigma_{k}(\tau)\right)=P_{0}$ and $G\left(\sigma_{k}(\tau)\right)=X\left(\sigma_{k}(0)\right)=0$. Moreover, our approach takes into account information about the parameter derivative, which is not considered in [15] and [16]. Then, as expected, our results lead to significantly better performance.

\subsection{Example 3}

Consider a VTOL (Vertical Take-off and Landing) helicopter linearized dynamic model described in $[12,33]$. This system can be represented by an LPV model (1) with the following matrices

$$
A(\sigma(t))=\left[\begin{array}{cccc}
-0.037 & 0.027 & 0.019 & -0.455 \\
0.048 & -1.010 & 0.002 & -4.021 \\
0.100 & \sigma(t) & -0.707 & 1.420 \\
0.000 & 0.000 & 1.000 & 0.000
\end{array}\right], \quad B=\left[\begin{array}{cc}
0.442 & 0.176 \\
3.545 & -7.592 \\
-5.520 & 4.490 \\
0.000 & 0.000
\end{array}\right]
$$

where the components of the state vector $x(t)$ represent the horizontal and vertical velocities (knots), the pitch rate (degrees/s) and the pitch angle (degrees). The input vector $u(t)$ corresponds to the collective pitch control and the longitudinal cyclic pitch control. The normalized control are given by $\bar{u}=\left[\begin{array}{ll}1 & 1\end{array}\right]^{\prime}$. The time-varying parameter $\sigma(t)=0.318+(\sin (2 t))^{2}$ is assumed to belong to the interval $[0.318,1.318]$ with $|\dot{\sigma}(t)| \leq 2$. In this example, we consider $\mathscr{T}_{1}=0.10 \mathrm{~s}, \varepsilon=2.5$, $Z=I$ and $H=0.01 I$. First, we analyze the impact of the intersampling upper bound $\mathscr{T}_{2}$ on the performance. For this, we consider the initial condition $x(0)=\left[\begin{array}{llll}1-2 & -4 & 3\end{array}\right]^{\prime}$. In this case, the maximum value of $\mathscr{T}_{2}$ for which it is possible to ensure the feasibility of $(56)$ is $0.95 \mathrm{~s}$. Furthermore, Table 5 shows the minimum values of $\gamma_{0}$ obtained for different values of $\mathscr{T}_{2}$, considering two different sets of constraints in the optimization problem (56): a) the ones obtained with $V$ and $\mathscr{V}_{0}$ being parameter-dependent (Theorem 2); and b) obtained with $V$ and $\mathscr{V}_{0}$ being parameter-independent (as commented in Remark 3). Observe that, although the use of parameter-dependent functionals increases the number of variables and computational complexity, smaller values of $\gamma_{0}$ and feasible results for greater values of $\mathscr{T}_{2}$ can be obtained. It can also be observed that, as $\mathscr{T}_{2}$ increases, the performance tends to degrade, i.e. $\gamma_{0}$ increases. This can also be seen by simulation in Figure 7 , where we compare the time responses considering $\mathscr{T}_{2}=0.2$ (solid line) and $\mathscr{T}_{2}=0.95$ (dash-dotted line). Note that the computed control signals $u_{1}$ and $u_{2}$ effectively saturate at the first instants of the simulation (the horizontal lines denote the control bounds).

We consider now the problem of maximizing the estimate of the region of attraction of the origin of the closed-loop system under the LPV-MPC strategy through the solution of optimization problem (63). The obtained values of $\alpha$, for $\mathscr{T}_{1}=0.01$ and different values of $\mathscr{T}_{2}$, considering again the parameter-dependent and independent cases, are shown in Table 6 . It can be seen that larger values of $\mathscr{T}_{2}$ result in larger values of $\alpha$. This means that increasing $\mathscr{T}_{2}$ leads to smaller sets of admissible initial conditions, for which is possible to ensure the LPV-MPC algorithm feasibility and the asymptotic convergence of the trajectories to the origin. Moreover, as expected, less conservative results (i.e. smaller values for $\alpha$ ) are obtained with the conditions derived from the parameter-dependent function and looped-functional. 


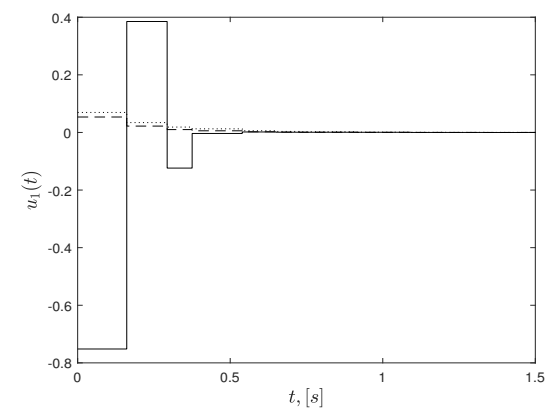

(a) Control input $u_{1}(t)$.

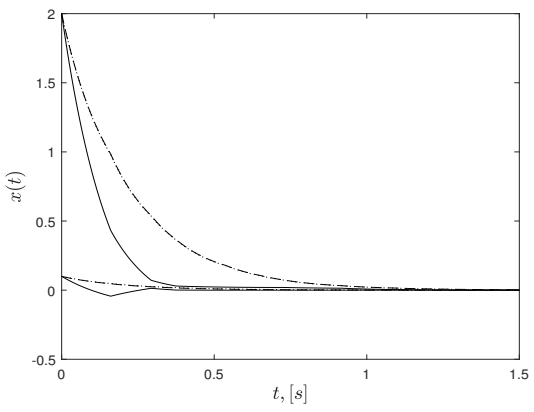

(c) Trajectories of the state vector $x(t)$.

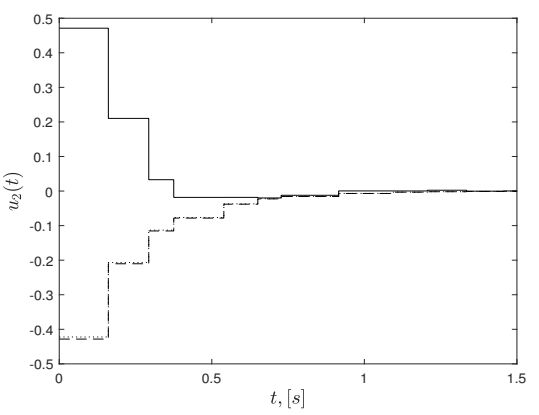

(b) Control input $u_{2}(t)$.

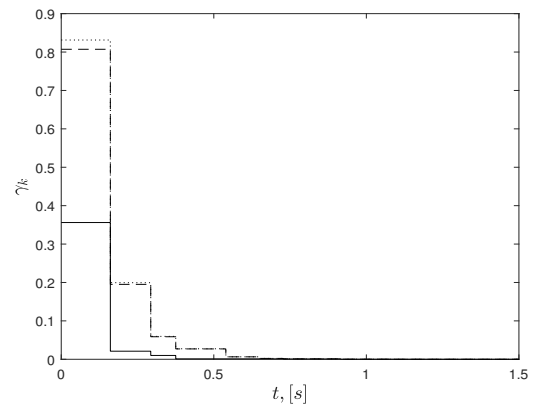

(d) Values of $\gamma(t)$.

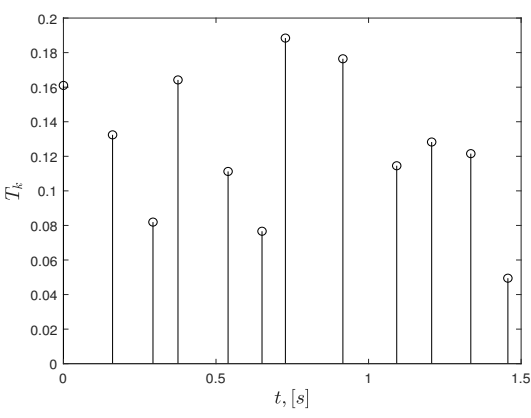

(e) Values of $T_{k}$.

Figure 6: Example 2 - Simulation results for $x(0)=[0.12]^{\prime}$ and $T_{k} \in[10,200] \mathrm{ms}$, from Algorithm 1 (solid line) and from algorithms in [16] (dotted line) and in [15] (dashed line).

Finally, we address the optimization problem (64), i.e. considering given $\mathscr{T}_{1}$ and a set $\mathscr{E}(\alpha I, 1)$ of admissible initial conditions, the aim is to maximize the upper bound $\mathscr{T}_{2}$ on the intersampling time. Table 7 shows the maximum value of $\mathscr{T}_{2}$ obtained for different values of $\alpha$, considering again the parameter-dependent and independent cases and $\mathscr{T}_{1}=0.10 \mathrm{~s}$. Note that for larger values of $\alpha$, i.e. for smaller pre-specified sets of admissible initial conditions for which we want to ensure stability of the origin, the values obtained for the maximum allowable value of $\mathscr{T}_{2}$ are larger, as expected. 


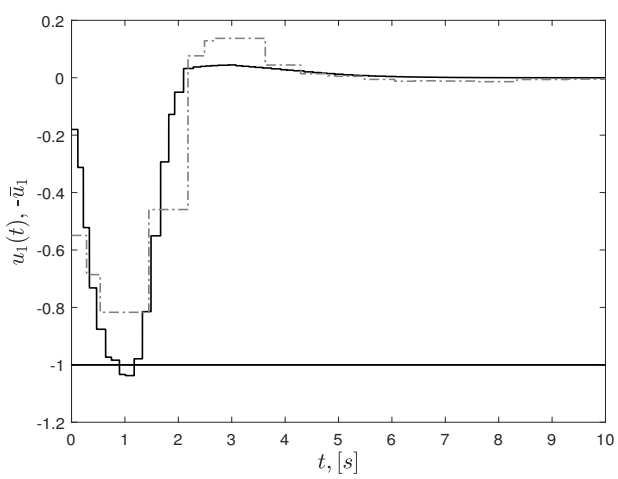

(a) Control input $u_{1}(t)$ and bound $\bar{u}$.

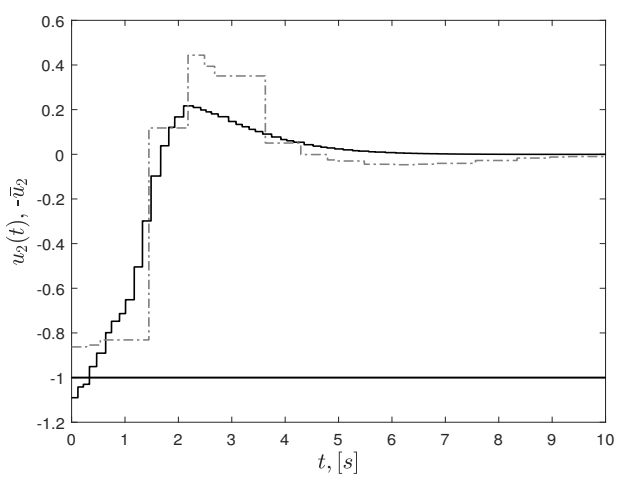

(b) Control input $u_{2}(t)$ and bound $\bar{u}$.

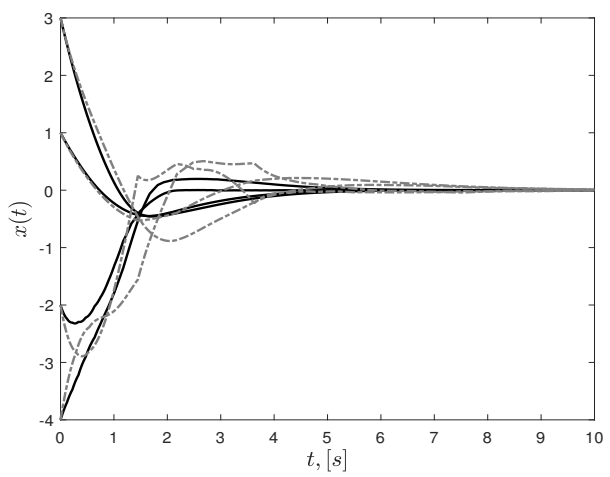

(c) Trajectories of the state vector $x(t)$.

Figure 7: Example 3 - Simulation results for $x(0)=\left[\begin{array}{llll}1 & -2 & -4 & 3\end{array}\right]^{\prime}$, considering $T_{k} \in\left[\begin{array}{lll}0.10, & 0.20\end{array}\right] \mathrm{s}$ (solid line) and $T_{k} \in[0.10,0.95] \mathrm{s}$ (dash-dotted line).

Table 5: Example 3 - Values of $\gamma_{0}$, for different values of $\mathscr{T}_{2}$, considering $\mathscr{T}_{1}=0.10$ s and $x(0)=\left[\begin{array}{llll}1 & -2 & -4 & 3\end{array}\right]^{\prime}$.

\begin{tabular}{ccc}
\hline $\mathscr{T}_{2}[\mathrm{~s}]$ & Parameter-dependent & Parameter-independent \\
\hline 0.10 & 135.9174 & 165.1483 \\
\hline 0.20 & 142.0219 & 173.0772 \\
\hline 0.50 & 147.5659 & 268.2552 \\
\hline 0.95 & 898.0019 & infeasible \\
\hline
\end{tabular}

\section{Concluding Remarks}

In this paper, the problem of sampled-data LPV-MPC state feedback control design has been addressed. The control signal has been assumed to be constant between two successive sampling instants and the continuous behavior of the LPV plant has been explicitly considered. Differently from previous approaches in the literature, the scheduling parameter is not supposed to be constant 
Table 6: Example 3 - Values of $\alpha$, for different values of $\mathscr{T}_{2}$ and conditions, considering $\mathscr{T}_{1}=0.10 \mathrm{~s}$.

\begin{tabular}{ccc}
\hline $\mathscr{T}_{2}[\mathrm{~s}]$ & Parameter-dependent & Parameter-independent \\
\hline 0.10 & 0.0990 & 0.1015 \\
\hline 0.20 & 0.0992 & 0.1029 \\
\hline 0.50 & 0.1042 & 0.1124 \\
\hline 0.95 & 0.1344 & 0.1712 \\
\hline
\end{tabular}

Table 7: Example 3 - Values of $\mathscr{T}_{2}(\mathrm{~s})$, for different values of $\alpha$ and conditions, considering $\mathscr{T}_{1}=0.10 \mathrm{~s}$.

\begin{tabular}{ccc}
\hline$\alpha$ & Parameter-dependent & Parameter-independent \\
\hline 0.095 & 1.22 & 0.80 \\
\hline 0.1 & 1.25 & 0.86 \\
\hline 0.15 & 1.41 & 1.10 \\
\hline 0.20 & 1.47 & 1.17 \\
\hline
\end{tabular}

between two successive sampling instants. Considering a polytopic approach that takes into account the bounds on magnitude and derivative of the scheduling parameters, an LPV system is used as a prediction model. Based on a parameter-dependent looped-functional to take into account the aperiodic sampling effects and a parameter-dependent generalized sector condition to tackle control saturation, conditions have been derived to the stabilization of the LPV system, such that an upper bound on a quadratic cost function is ensured. From these conditions, an MPC based algorithm has been proposed to compute an LPV control law at each sampling instant considering the sampled values of states and parameters, with the aim of minimizing the upper bound on the cost function. To do so, an LMI-based optimization problem is solved. It has been explicitly proven that the proposed scheme ensures the asymptotic convergence to the origin, provided the initial state belongs to a certain set in state space. Based on this LMI formulation, and differently from previous works, an explicit characterization of this set, which can also be seen as an estimate of the region of attraction of the origin, is provided. The fact of considering a parameter-dependent loopedfunctional and also a less conservative polytopic modeling, which takes into account the bounds on the parameters derivatives, leads to less conservative results (both in terms of performance and the size of the set of admissible initial conditions) when compared to similar approaches in the literature, as shown in numerical examples. The numerical complexity of the LMI problem to be solved at each sampling instant depends on the system dimensions. In cases where the computation time is critical, a trade-off between conservatism and complexity can be considered by using an alternative formulation based on parameter-independent conditions.

The method can be applied to a broad class of systems that inherently present uncertain and time-varying parameters or nonlinear systems that can be represented by a quasi-LPV model, i.e. when some continuous nonlinearities can be converted to state-dependent parameters and the system cast as in (1).

Future work may address the extension of the proposed sampled-data MPC strategy to some specific classes of nonlinear systems, such as rational or Lur'e ones, whose stabilizing conditions can be cast in an LMI framework (similar to the one considered here for LPV systems) and the design of output feedback control laws. A special interest in this case arises from applications in robotics and 
unmanned autonomous vehicles [34, 35]. In particular the way of dealing with arbitrary references is a special challenge. Moreover, the use of LPV models to represent autonomous vehicles should be carefully evaluated.

Some other interesting future research directions regards the extension of the proposed approach to consider stochastic aspects, the presence of delays [36,37] and also the synchronization of systems networks [38] using the MPC approach and LMI methods. Although the case of asymmetric saturation can be treated with the proposed results, by considering a worst case symmetric scenario, this is also an open issue to be considered.

\section{Acknowledgments}

This study was financed in part by the Coordenação de Aperfeiçoamento de Pessoal de Nível Superior - Brazil (CAPES) - Finance Code 001 (PROEX and STICAmSud programs) and by the Conselho Nacional de Desenvolvimento Científico e Tecnológico - Brazil (CNPq) - Grants $422992 / 2016-0,306223 / 2018-0$ and 307449/2019-0.

\section{References}

[1] M. V. Kothare, V. Balakrishnan, M. Morari, Robust constrained model predictive control using linear matrix inequalities, Automatica 32 (10) (1996) 1361-1379.

[2] C. E. Garcia, D. M. Prett, M. Morari, Model predictive control: theory and practice - a survey, Automatica 25 (3) (1989) 335-348.

[3] S. J. Qin, T. A. Badgwell, A survey of industrial model predictive control technology, Control Engineering Practice 11 (7) (2003) 733-764.

[4] E. F. Camacho, C. B. Alba, Model predictive control, Springer, London, 2013.

[5] J. M. Maciejowski, Predictive control with constraints, Prentice Hall, Englewood Cliffs, 2002.

[6] C. Briat, Linear parameter-varying and time-delay systems: Analysis, observation, filtering \& control, Vol. 3 of Advances in Delays and Dynamics, Springer, Heidelberg, Germany, 2015.

[7] J. Mohammadpour, C. Scherer (Eds.), Control of Linear Parameter Varying Systems with Applications, Springer, New York, 2012.

[8] A. Casavola, D. Famularo, G. Franze, Predictive control of constrained nonlinear systems via LPV linear embeddings, International Journal of Robust and Nonlinear Control 13 (3-4) (2003) $281-294$.

[9] N. Wada, K. Saito, M. Saeki, Model predictive control for linear parameter varying systems using parameter dependent Lyapunov function, in: 47th International Midwest Symposium on Circuits and Systems, Vol. 3, IEEE, 2004, pp. 133-136.

[10] A. Casavola, D. Famularo, G. Franze, E. Garone, An improved predictive control strategy for polytopic LPV linear systems, in: 45th IEEE Conference on Decision and Control, IEEE, 2006, pp. $5820-5825$. 
[11] M. Jungers, R. C. d. L. F. Oliveira, P. L. D. Peres, MPC for LPV systems with bounded parameter variations, International Journal of Control 84 (1) (2011) 24-36.

[12] M. F. Braga, C. F. Morais, E. S. Tognetti, R. C. Oliveira, P. L. D. Peres, Discretization and event triggered digital output feedback control of LPV systems, Systems \& Control Letters 86 (2015) 54-65.

[13] W. Zhang, M. Branicky, S. Phillips, Stability of Networked Control Systems, IEEE Control Systems Magazine 21(1) (2001) 84-99.

[14] L. Hetel, C. Fiter, H. Omran, A. Seuret, E. Fridman, J.-P. Richard, S. I. Niculescu, Recent developments on the stability of systems with aperiodic sampling: An overview, Automatica 76 (2017) 309-335.

[15] T. Shi, H. Su, Sampled-data MPC for LPV systems with input saturation, IET Control Theory \& Applications 8 (17) (2014) 1781-1788.

[16] T. Raff, D. Sinz, F. Allgower, Model predictive control of uncertain continuous-time systems with piecewise constant control input: a convex approach, in: 2008 American Control Conference, IEEE, 2008, pp. 1109-1114.

[17] E. Fridman, A. Seuret, J.-P. Richard, Robust sampled-data stabilization of linear systems: An input delay approach, Automatica 40 (8) (2004) 1141-1446.

[18] E. Fridman, A refined input delay approach to sampled-data control, Automatica 46 (2) (2010) 421-427.

[19] J. P. Hespanha, P. Naghshtabrizi, Y. Xu, A survey of recent results in networked control systems, Proceedings of the IEEE 95 (1) (2007) 138-162.

[20] D. Li, Y. Xi, P. Zheng, Constrained robust feedback model predictive control for uncertain systems with polytopic description, International Journal of Control 82 (7) (2009) 1267-1274.

[21] C. Briat, A. Seuret, A looped-functional approach for robust stability analysis of linear impulsive systems, Systems \& Control Letters 61 (10) (2012) 980-988.

[22] A. Seuret, A novel stability analysis of linear systems under asynchronous samplings, Automatica 48 (1) (2012) 177-182.

[23] J. M. Gomes da Silva Jr., A. H. K. Palmeira, V. M. Moraes, J. V. Flores, L2-disturbance attenuation for LPV systems under sampled-data control, International Journal of Robust and Nonlinear Control 28 (16) (2018) 5019-5032.

[24] J. M. Gomes da Silva Jr., S. Tarbouriech, Antiwindup design with guaranteed regions of stability: an LMI-based approach, IEEE Transactions on Automatic Control 50 (1) (2005) $106-111$.

[25] J. M. Gomes da Silva Jr., V. M. Moraes, J. V. Flores, A. H. K. Palmeira, Sampled-data LPV control: A looped functional approach, IFAC-PapersOnLine 48 (2015) 19-24.

[26] S. Tarbouriech, G. Garcia, J. M. Gomes da Silva Jr., I. Queinnec, Stability and Stabilization of Linear Systems with Saturating Actuators, Springer, 2011. 
[27] A. Seuret, J. M. Gomes da Silva Jr., Taking into account period variations and actuator saturation in sampled-data systems, Systems \& Control Letters 61 (12) (2012) 1286-1293.

[28] A. H. K. Palmeira, J. M. Gomes da Silva Jr., J. V. Flores, Aperiodic sampled-data control for LPV systems under input saturation, IFAC-PapersOnLine 51 (2018) 130-136.

[29] M. Jungers, E. B. Castelan, Gain-scheduled output control design for a class of discrete-time nonlinear systems with saturating actuators, Systems \& Control Letters 60 (3) (2011) 169-173.

[30] C. Briat, Convergence and equivalence results for the Jensen's inequality - application to timedelay and sampled-data systems, IEEE Transaction on Automation Control 56 (7) (2011) $1660-1665$.

[31] H. D. Tuan, P. Apkarian, T. Narikiyo, Y. Yamamoto, Parameterized linear matrix inequality techniques in fuzzy control system design, IEEE Transactions on fuzzy systems 9 (2) (2001) $324-332$.

[32] Z. Wan, M. V. Kothare, An efficient off-line formulation of robust model predictive control using linear matrix inequalities, Automatica 39 (5) (2003) 837-846.

[33] L. H. Keel, S. Bhattacharyya, J. W. Howze, Robust control with structure perturbations, IEEE Transactions on Automatic Control 33 (1) (1988) 68-78.

[34] E. Alcalá, V. Puig, J. Quevedo, LPV-MPC control for autonomous vehicles, IFACPapersOnLine 52 (28) (2019) 106-113.

[35] Z. Yan, P. Gong, W. Zhang, W. Wu, Model predictive control of autonomous underwater vehicles for trajectory tracking with external disturbances, Ocean Engineering 217 (2020) 107884:110.

[36] G. Zhang, Q. Zhu, Guaranteed cost control for impulsive nonlinear Itô stochastic systems with mixed delays, Journal of the Franklin Institute 357 (2020) 6721-6737.

[37] Q. Zhu, Stabilization of stochastic nonlinear delay systems with exogenous disturbances and the event-triggered feedback control, IEEE Transactions on Automatic Control 64 (9) (2019) $3764-3771$.

[38] Y. Wu, Y. Lu, S. He, R. Lu, Synchronization control for unreliable network systems in intelligent robots, IEEE/ASME Transactions on Mechatronics 24 (6) (2019) 2641-2651. 